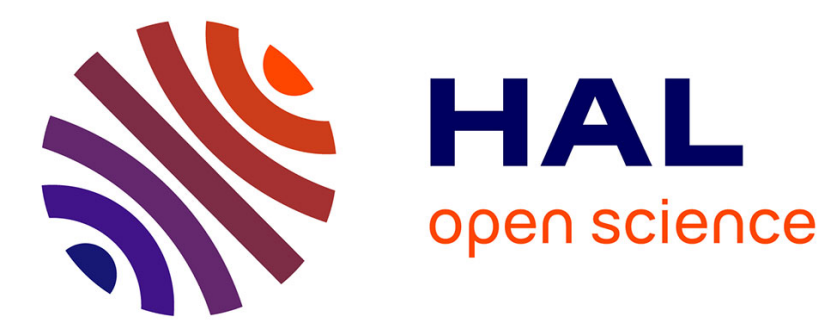

\title{
The interior structure of Mercury and its core sulfur content
}

\author{
A. Rivoldini, T. van Hoolst, O. Verhoeven
}

\section{To cite this version:}

A. Rivoldini, T. van Hoolst, O. Verhoeven. The interior structure of Mercury and its core sulfur content. Icarus, 2009, 201 (1), pp.12. 10.1016/j.icarus.2008.12.020 . hal-00524850

\section{HAL Id: hal-00524850 \\ https://hal.science/hal-00524850}

Submitted on 9 Oct 2010

HAL is a multi-disciplinary open access archive for the deposit and dissemination of scientific research documents, whether they are published or not. The documents may come from teaching and research institutions in France or abroad, or from public or private research centers.
L'archive ouverte pluridisciplinaire HAL, est destinée au dépôt et à la diffusion de documents scientifiques de niveau recherche, publiés ou non, émanant des établissements d'enseignement et de recherche français ou étrangers, des laboratoires publics ou privés. 


\section{Accepted Manuscript}

The interior structure of Mercury and its core sulfur content

A. Rivoldini, T. Van Hoolst, O. Verhoeven

PII:

S0019-1035(08)00448-X

DOI: $\quad$ 10.1016/j.icarus.2008.12.020

Reference: $\quad$ YICAR 8858

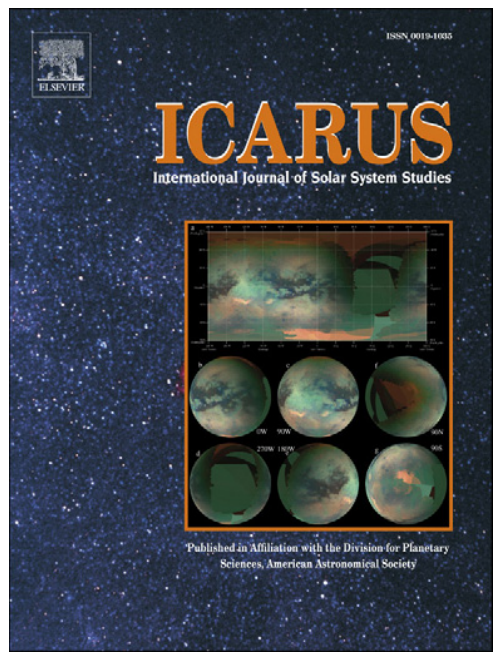

To appear in: Icarus

Received date: 24 March 2008

Revised date: 14 November 2008

Accepted date: 15 December 2008

Please cite this article as: A. Rivoldini, T. Van Hoolst, O. Verhoeven, The interior structure of Mercury and its core sulfur content, Icarus (2009), doi: 10.1016/j.icarus.2008.12.020

This is a PDF file of an unedited manuscript that has been accepted for publication. As a service to our customers we are providing this early version of the manuscript. The manuscript will undergo copyediting, typesetting, and review of the resulting proof before it is published in its final form. Please note that during the production process errors may be discovered which could affect the content, and all legal disclaimers that apply to the journal pertain. 


\title{
The interior structure of Mercury and its core sulfur content
}

\author{
A. Rivoldini, T. Van Hoolst, O. Verhoeven, \\ Royal Observatory of Belgium, Avenue Circulaire 3, B-1180 Brussels, Belgium
}

Number of manuscript pages: 67

Number of manuscript tables: 3

Number of manuscript figures: 18 
Proposed running head: Interior structure of Mercury

Editorial Correspondence to:

Attilio Rivoldini

Royal Observatory of Belgium

3, avenue Circulaire

B-1180 Brussels

Belgium

Email: Attilio.Rivoldini@oma.be

Phone: +322 3736726

Fax: +3223749822 


\section{Abstract}

Depth-dependent interior structure models of Mercury are calculated for several plausible chemical compositions of the core and of the mantle. For those models, we compute the associated libration amplitude, obliquity, tidal deformation, and tidal changes in the external potential.

In particular we study the relation between the interior structure parameters for five different mantle mineralogies and two different temperature profiles together with two extreme crust density values. We investigate the influence of the core light element concentration, temperature, and melting law on core state and inner and outer core size. We show that a sulfur concentration above $10 \mathrm{wt} \%$ is unlikely if the temperature at the core mantle boundary is above $1850 \mathrm{~K}$ and the silicate shell at least $240 \mathrm{~km}$ thick. The interior models can only have an inner core if the sulfur weight fraction is below $5 \mathrm{wt} \%$ for core mantle boundary temperature in the $1850 \mathrm{~K}-2200 \mathrm{~K}$ range.

Within our modeling hypotheses, we show that with the expected precision on the moment of inertia the core size can be estimated to a precision of about $50 \mathrm{~km}$ and the core sulfur concentration with an error of about $2 \mathrm{wt} \%$. This uncertainty can only be reduced when more information on the mantle mineralogy of Mercury becomes available. However, we show that the uncertainty on the core size estimation can be greatly reduced, to about $25 \mathrm{~km}$, if tidal surface displacements and tidal variations in the external potential are considered.

Key words: Mercury, interiors, rotational dynamics, solid body tides, spin-orbit resonances 


\section{Introduction}

After more than 30 years without observations from a close spacecraft, three spacecraft will extensively investigate Mercury in the next decade. The NASA MESSENGER mission will start its yearlong observations from orbit around Mercury in 2011 and has already made first measurements during its first flyby in January 2008. The BepiColombo mission to Mercury of ESA and JAXA is scheduled for launch in 2013 and is foreseen to start its science phase from orbit in 2019. The mission consists of two orbiting spacecraft, the Mercury Planetary Orbiter (MPO), intended primarily for an in-depth investigation of Mercury's interior and surface, and the Mercury Magnetospheric Orbiter (MMO), which will focus on the characterization of Mercury's magnetosphere. Both missions will help unlocking Mercury's interior. Here, we assess the improvements on the deep interior structure expected from geodetic spacecraft observations.

The interior structure of Mercury is the least known of the four terrestrial planets. The main knowledge can be summarized as follows. The relatively large mass of $m_{a}=3.302 \times 10^{23} \mathrm{~kg}$ for its small radius of $r_{a}=2439 \pm 1 \mathrm{~km}$ (Anderson et al., 1987) indicates that Mercury has a large core. If the core consists mainly of iron, the core radius will be about $3 / 4$ of the radius of the planet, which is relatively larger than for the three other terrestrial planets. Thermal evolution models (Schubert et al., 1988; Hauck et al., 2004; Breuer et al., 2007) suggest that the core is divided into a solid inner core and a liquid outer core like the Earth. The magnetic field observed by Mariner 10, if created by a dynamo, provides evidence for a liquid outer core and is also a strong indication for a solid inner core. Additional evidence for a, at least, partially liquid outer core is provided by recent radar measurements by Margot et al. 
(2007), which show that the surface librations are larger than would be for an entirely solid planet.

Since the earliest models of Mercury's interior, the only global geophysical constraints are the mass and radius (or average density $\bar{\rho}=5434 \mathrm{~kg} / \mathrm{m}^{3}$ ). Plausible compositions are inferred from cosmochemical considerations on chemical element distribution in the solar nebula and from hypotheses on Mercury's formation. The older models used only iron in the core (Siegfried and Solomon, 1974) based on the erroneous assumption that Mercury formed from solar nebula material close to Mercury's actual distance from the Sun and should therefore be depleted in volatiles. More recent models also incorporate the light element sulfur in the core (e.g. Harder and Schubert, 2001). With an iron-sulfur core, the possible core size ranges form $72 \%$ to almost $100 \%$ of the planet's radius (Harder and Schubert, 2001). A possible inner core has been considered by Spohn et al. (2001) and Van Hoolst and Jacobs (2003) for models with homogeneous layers (inner core, outer core, and mantle). The inner core is assumed to consist of pure, solid $\gamma-\mathrm{Fe}$ and the larger the inner core grows the more the liquid iron core becomes enriched in sulfur. Hauck et al. (2007) also used a constant-density silicate shell, but developed a more detailed and accurate model of the core by using data on the liquid iron-sulfur system for the liquid outer core.

Here, our goal is twofold: first, we construct accurate models of the whole deep interior of Mercury and secondly, we study the expected improvements on Mercury's interior from future geodetic spacecraft and Earth-based observations. Whereas previous models used simplifying assumptions on the composition or structure either for the core or for the mantle and crust, we here address both the silicate shell and the dominantly iron core. Following Verhoeven et al. 
(2008) we here determine mineralogical models of the mantle of Mercury for several of the compositional models of Taylor and Scott (2005), and we calculate radial profiles of density, pressure and seismic wave velocities. The core is assumed to consist of iron and sulfur and we include the most recent data on thermo-elastic properties of the relevant liquid metal alloys and their melting properties. We calculate density, pressure, and temperature profiles in the core for mantle temperature profiles determined in studies on the evolution of Mercury (Spohn, 1991; Buske, 2006; Breuer et al., 2007). The inner core size is determined by requiring the temperature at the boundary between the solid inner core and the liquid outer core to be equal to the melting temperature of the outer core.

Future spacecraft missions together with Earth-based radar observations are expected to constrain the internal structure of Mercury by determining its gravity field, tides, libration amplitude and obliquity with unprecedented accuracy (e.g. Van Hoolst et al. (2007); Zuber et al. (2007)). The rotation quantities (libration and obliquity) will determine the moment of inertia of Mercury and the moment of inertia of the silicate shell with a precision on the order of a percent. The moment of inertia will constrain the size and the composition of the core, but without any further data it can only slightly improve the estimation on the core size and sulfur content of current models because of uncertainties in the mantle composition and the inner core size (Harder and Schubert, 2001; Spohn et al., 2001; Van Hoolst and Jacobs, 2003). The mantle moment of inertia is more sensitive to the size and density of the core and therefore could constrain the core better, although it doesn't permit to estimate the inner core size (Van Hoolst et al., 2007). From a large set of about 800,000 models, Hauck et al. (2007) estimated that the size and density of the core and 
the mean density of the silicate shell can be determined to within $\pm 23 \mathrm{~km}$, $\pm 147 \mathrm{~kg} / \mathrm{m}^{3}$, and $\pm 72 \mathrm{~kg} / \mathrm{m}^{3}$, respectively, from MESSENGER observations. Further insight into the interior of Mercury can be obtained by studying Mercury's tides due to the Sun. Both the tidal surface displacements and the tidal changes in external gravity field variations allow tightly constraining the inner and outer core (Spohn et al., 2001; Van Hoolst and Jacobs, 2003). For the first time, we here perform a full analysis of the geodesy constraints on detailed models of Mercury's interior. In particular, tides have only been studied by Spohn et al. (2001) and Van Hoolst and Jacobs (2003), but for simplified models with core and mantle considered as layers of constant density.

The paper is organized as follows. In Sect. 2, we describe the composition models for the mantle and the core of our Mercury models. Section 3 starts with an overview of the governing equations and the mathematical method used to calculate the interior structure models. In particular in Sect. 3.2, we discuss the equations of state for the core. The resulting models are discussed in Section 3.3. Section 4 is devoted to an analysis of the moments of inertia and tides of the Mercury models. A discussion and conclusions are presented in the final section.

\section{Composition}

The large core of Mercury implies large differences in bulk composition with respect to the other terrestrial planets, in particular a much larger metalto-silicate ratio of more than 1 . Classical equilibrium condensation accretion models in which planets form by condensation from the local solar nebula yield a maximum relative iron core mass of about 36\% (Lewis, 1988), below 
the Mercury value. Therefore, Mercury must have had an unusual formation history. A scenario in which Mercury suffered a collision with another large proto-planet (Benz et al., 1988; Wetherill, 1988) is currently considered the most likely (e.g. Taylor and Scott, 2005), but other scenarios exist such as condensation models in which metal and silicate grains were aerodynamically sorted (Weidenschilling, 1978) or partial vaporization of the outer silicate shell after formation (Cameron, 1985). The giant impact model assumes that much of the silicate failed to reaccrete, which requires a high impact velocity and, therefore, a collision close to the Sun such as at the current distance of Mercury (Benz et al., 2007). This results in an almost completely destroyed planet and to extremely high temperatures leading to the loss of most of the volatile components (Benz et al., 2007). Obviously, different formation histories lead to different chemical compositions.

\subsection{Mantle and crust}

The only observation constraining the mantle composition is the spectroscopic determination of the low $\mathrm{FeO}$ content $\lesssim 3$ wt\% of the surface of Mercury Sprague et al. (1994); Jeanloz et al. (1995); Boynton et al. (2007). Since many smooth plains with low FeO content have morphological features consistent with a lava flow origin and the FeO content of lava is considered to be close to that of the mantle source region, Robinson and Taylor (2001) concluded that Mercury's mantle is equally low in FeO. Several geochemical models for Mercury's mantle have been designed that satisfy this constraint (Taylor and Scott, 2005). Among the three different formation scenarios discussed above, only for the vaporization of the outer silicate shell 
detailed models of the composition of Mercury's silicate part have been developed (Taylor and Scott, 2005; Benz et al., 2007). Other models with FeO content $\lesssim 3$ wt\% either use a mixture of refractory and volatile materials (Morgan and Anders, 1980; Taylor and Scott, 2005) or consider specific chondrites as building blocks for Mercury silicate part (Krot et al., 2001; Wasson, 1988) Based on a thermodynamic model of mineral phase equilibria as a function of pressure and temperature, Verhoeven et al. (2008) have computed the mantle mineralogy for several of such models for two end-member temperature profiles. We here extend their calculations to a depth of $640 \mathrm{~km}$. These models are (see Figs. 1):

(1) model FC: the evaporation model of Fegley and Cameron (1987) assumes a rapid accretion of Mercury close to the Sun and a subsequent vaporization of $70-80 \%$ of silicate magma of chondritic composition. The main feature of this model is a strong depletion in $\mathrm{SiO}_{2}$ (19.9 wt\%), which induces a mineralogy dominated by oxides (spinel $\mathrm{MgAl}_{2} \mathrm{O}_{4}$ and periclase $\mathrm{MgO}$ form $50 \%$ of the mantle volume).

(2) model MA: the model of Morgan and Anders (1980) is based on the assumption that Mercury underwent fractionation processes in the solar nebula. By assuming core mass, FeO content, and Moon-like abundances of some radiogenic elements they deduce a mantle composition made of about $30 \%$ olivine, $50 \%$ pyroxenes and $20 \%$ garnet.

(3) Model TS: the refractory-volatile mixture of Taylor and Scott (2005) takes into account that planetary accretion involved mixing throughout the inner Solar System. The model is a mixture of an ultra-refractory Mercury (Goettel, 1988) and the Earth's primitive mantle that predicts a FeO content equal to 3wt\%. The mineralogy of model TS is dominated 
by olivine which accounts for about $60 \%$ of the mantle volume.

(4) model MC: the metal-rich chondrite model is based on the idea that primitive chondrules in two metal-rich chondrites (Krot et al., 2001) might be suitable Mercury building blocks (Taylor and Scott, 2005). Such chondrules are characterized by a low FeO content and formed under oxidizing conditions, above the metal condensation temperature. The mantle composition is very close to the composition of the MA model.

(5) model EC: the enstatite meteorite model is based on the assumption that such meteorites formed at the same location as Mercury (Wasson, 1988). Specific features of the EC mantle are the absence of olivine and the presence of about $15 \%$ coesite, a quartz polymorph. This mantle is also characterized by a phase transition (quartz-coesite) at a depth of about $240 \mathrm{~km}$.

Due to the small pressure in Mercury's mantle (from about 3GPa to 9GPa for a reasonable range of core sizes), major phase transitions as in the Earth's mantle from olivine to its high pressure phases wadsleyite and ringwoodite and the transition to perovskite and magnesiowüstite do not occur inside Mercury. The mineralogy in the mantle depends only weakly on temperature and pressure for depths less than about 500km, resulting in an almost homogeneous main part of the mantle (Figs. 1 and 2). Olivine, pyroxenes and garnet are the dominant minerals, except for the $\mathrm{SiO}_{2}$-poor mantle of Fegley and Cameron (1987), which contains more uncommon minerals like merwinite and periclase.

The FC mantle has the highest average density (about $3470 \mathrm{~kg} / \mathrm{m}^{3}$ ) due to the presence of the dense mantle minerals merwinite, periclase and spinel and the EC mantle the lowest density (about $3170 \mathrm{~kg} / \mathrm{m}^{3}$ ) because of the dominant presence of the low density minerals coesite and orthopyroxene (see Fig. 2). 
For the selected core size of $1790 \mathrm{~km}$ (depth of $650 \mathrm{~km}$ ), the pressure at the coremantle boundary varies between $8.2 \mathrm{GPa}$ and $8.6 \mathrm{GPa}$. The mantle density is only weakly dependent on temperature (Fig. 2) leading to relative differences in the average density between the individual cold and hot mantle models that are below $1 \%$.

Heat flux considerations at the base of the crust of Mercury and surface faulting observations require that the crust thickness is below 140km (Nimmo and Watters, 2004). Moreover, the stability of the long-wavelength topography on Mercury suggests that the crust thickness is below 115km (Grott and Breuer, 2007). For our modeling purposes we assume the crust to be $100 \mathrm{~km}$ thick. In principle, the crust composition can be calculated from the mantle mineralogy by assuming that the crust formed as a lava from the mantle. However, due to lack of experimental data on melting of $\mathrm{a} \mathrm{SiO}_{2}$-poor mantle, the computation of the lava crust composition is not possible for models FC and TS. Moreover, a regolith layer of about $10 \mathrm{~km}$ thick may be present in the Hermean crust (Breuer et al., 2007). Because of these uncertainties on the crust, we here consider a low- and a high-density crust with homogeneous densities of $2900 \mathrm{~kg} / \mathrm{m}^{3}$ and $3300 \mathrm{~kg} / \mathrm{m}^{3}$, and seismic velocities equal to $v_{p}=7900 \mathrm{~m} / \mathrm{s}$ and $v_{s}=4550 \mathrm{~m} / \mathrm{s}$. For the MC and EC mantles we only consider the low-density end-member crust as their density in the upper part of the mantle is below $3300 \mathrm{~kg} / \mathrm{m}^{3}$ (Fig. 2).

\subsection{Core}

The proximity of Mercury to the Sun does not necessarily imply that the core consists essentially of pure iron with almost no light elements included since 
Mercury formed from planetesimals originating from a wide zone covering a distance to the Sun between about the actual position of Mercury and the asteroid belt (Benz et al., 2007). Because the accretion process is basically a random phenomenon, the amount of light elements cannot be predicted, even if the formation model explaining the large core were to be known. Besides iron, the core of Mercury might also contain nickel up to $8 \mathrm{wt} \%$, which is the cosmic abundance of nickel. Possible light elements are S, Si, O, C, H..., in binary, ternary or more complicated systems with Fe and Ni.

Among the light elements sulfur, under the form of FeS, has been found in many nickel-iron-meteorites, and is therefore ubiquitously invoked as a major candidate for light elements in planetary cores of terrestrial planets. Of all the light elements, sulfur and silicon are most easily incorporated into planetary cores of terrestrial planets as their solubility in molten iron is high over an extended pressure range. Oxygen solubility in liquid iron is below $1 \mathrm{wt} \%$ at ambient pressure and, although increasing with increasing pressure, remains small and below about 1wt\% for pressures below 10GPa (Tsuno et al., 2007).

In ternary iron systems, the solubility of light elements is more complicated. For example, Si does not dissolve into liquid $\mathrm{Fe}-\mathrm{S}$ at ambient pressure, the first melt to percolate through the silicate matrix during core formation, since at that pressure the $\mathrm{Fe}-\mathrm{S}-\mathrm{Si}$ system exhibits a large region of immiscibility in a temperature composition representation. This miscibility gap only closes at about 14GPa (Sanloup and Fei, 2004). Since the differentiation of Mercury took place at pressures below $14 \mathrm{GPa}(\mathrm{CMB}$ pressures are about $5-8 \mathrm{GPa}), \mathrm{Si}$ could not have dissolved in the $\mathrm{Fe}-\mathrm{S}$ melt and it is therefore unlikely that significant amounts of Si are incorporated into the core. Oxygen, although almost immiscible with liquid iron at low pressure, could be dissolved in $\mathrm{Fe}-\mathrm{S}$ melts 
since the miscibility increases with increasing temperature, pressure and sulfur weight fraction (Tsuno et al., 2007). However, data in Tsuno et al. (2007) and Rubie et al. (2004) suggest that at the low pressures characteristic for Mercury's differentiation and the low sulfur concentration considered here $\left(x_{\mathrm{S}}<10 \mathrm{wt} \%\right)$ oxygen too can only be present in small amounts in Mercury's core. Significant amounts of O could only have dissolved into the core during the hot early period of the planets history if the initial sulfur abundance were sufficiently high.

As a consequence of the planet's cooling history, a solid inner core may have formed in the initial liquid core of Mercury. Light elements are not only dissolved in the liquid $\mathrm{Fe}-\mathrm{Ni}$ outer core mixture but can also be incorporated in the solid $\mathrm{Fe}-\mathrm{Ni}$ inner core alloy. The solubility of $\mathrm{Si}$ in solid Fe is nearly identical to its solubility in liquid Fe (Kuwayama and Hirose, 2004), whereas S only dissolves for less than about $1 \mathrm{wt} \%$ in solid Fe at the low core pressures in Mercury (Li et al., 2001). The solubility of S in solid Fe - Ni (Zhang and Fei, 2008) increases to more than $2 \mathrm{wt} \%$ depending on the amount of $\mathrm{Ni}$. The light elements S and Si also have the important property that they lower the melting temperature of the iron system with respect to pure iron, contrary to oxygen which does not significantly depress the melting temperature of $\mathrm{Fe}$ (Boehler, 1992; Tsuno et al., 2007). For example, at 21GPa the melting temperature of Fe decreases by about $60 \mathrm{~K}$ for each additional weight percent of $\mathrm{S}$ (Fei et al., 2000) and the temperature decrease for the inclusion of Si is about 10K (Kuwayama and Hirose, 2004). Note that the melting temperature decreases with increasing amounts of nickel (Stewart et al., 2007; Zhang and Fei, 2008) (for about $8 \mathrm{wt} \%$ of $\mathrm{Ni}$ at $23 \mathrm{GPa}$ it is about $30 \mathrm{~K}$ ).

The above data for binary and ternary iron systems suggest that it is unlikely 
that Mercury's core contains significant amounts of light elements other than sulfur. Moreover, sulfur is the only light element that strongly reduces the melting temperature of the iron-rich outer core. This might be needed for a liquid outer core to exist in Mercury. Therefore, we here assume that sulfur is the only light element in Mercury's core. There is however a caveat, the pressure inside Mercury could have been higher if the initial proto-planet was larger, e.g. prior to the outer silicate shell vaporization/abrasion or in the event of a giant impact. The pressure and temperature conditions could have been high enough to favor inclusion of the light elements $\mathrm{Si}$ and $\mathrm{O}$ beside $\mathrm{S}$ in the forming core. On the other hand, the extreme heating associated with the event of a giant impact results in the loss of most of the volatile light elements and suggests that the reforming core will essentially be made of the heaviest elements $(\mathrm{Fe}, \mathrm{Ni})$.

Inner-core growth by solid-iron precipitation at the inner core boundary starts when the local core temperature drops below the local liquidus temperature if the sulfur weight fraction is to the left of the eutectic composition. At the typical pressures and temperatures of Mercury's core, solid iron is in the fcc phase ( $\gamma$ iron, Ahrens et al., 2002). Solid sulfur can occur in different ironsulfide phases (FeS IV and V) and compounds ( $\mathrm{FeS}, \mathrm{Fe}_{3} \mathrm{~S}_{2}, \mathrm{Fe}_{3} \mathrm{~S}$ and $\mathrm{Fe}_{2} \mathrm{~S}$, see Fei et al., 1995, 1997, 2000), but we do not include these solids since the strong partitioning of sulfur in the liquid phase results in an inner core made of almost pure $\gamma-$ Fe. Therefore, the liquid outer core will gradually increase its light element concentration until it attains the eutectic composition, which is characterized by a sharp minimum in the liquidus curve. Upon further cooling, the outer core liquid gradually freezes, and solids of eutectic composition are deposited onto the solid inner core. On the other hand if the initial core sulfur 
weight fraction is above the eutectic composition, solids rich in $\mathrm{S}$ will crystallize out of the liquid where the local temperature drops below the liquidus. Depending on the pressure and temperature conditions, this sulfur-rich component will either result in a growing inner core or a deposition of a light element rich layer at the core-mantle boundary (Hauck et al., 2006). The S fraction in the remaining liquid will gradually decrease until the eutectic concentration and gradually freezes out upon further cooling. Here, we only consider a small amount of sulfur to be able to keep an appreciable silicate shell of at least $240 \mathrm{~km}$ thick. With this choice, the sulfur concentration of the core is smaller than 10wt\% (See Section 2) and at the left side of the eutectic.

In order to obtain an empirical melting law valid for the core material we have fitted the liquidus data relevant to Mercury's core on melting data of $\mathrm{Fe}-\mathrm{S}$ at $(10,14,21,23,25,40) \mathrm{GPa}$ (Fei et al., 1997; Li et al., 2001; Stewart et al., 2007) for $x_{S} \leq 0.1$ and melting data of pure Fe (Boehler, 1996; Shen et al., 1998) on the following expression

$$
T_{m}(r)=T_{m 0}\left(1-\alpha_{c} x_{s}-\beta_{c} x_{s}^{2}\right)\left(1+T_{m 1} P(r)+T_{m 2} P^{2}(r)\right) .
$$

We have $\alpha_{c}=0.5419, \beta_{c}=13.583, T_{m 0}=1820.9 K, T_{m 1}=0.01402 \mathrm{~Pa}^{-1}$, $T_{m 2}=0.0001 \mathrm{~Pa}^{-2}$. This melting law quadratic in $P$ and $x_{\mathrm{S}}$ is a straightforward generalization of the customarily used melting law linear in $x_{S}$ and quadratic in $P$ (e.g. Stevenson et al., 1983; Hauck et al., 2004). It is a first attempt to include the non-linear dependence of the melting temperature on $x_{\mathrm{S}}$ at constant pressure, which is clearly visible in any $\mathrm{Fe}-\mathrm{S}$ phase diagram. 


\section{$3 \quad$ Interior structure}

\subsection{Method}

We construct spherically-symmetric models of the interior structure of Mercury in which interior structure quantities only depend on the radial distance to the center $r$. We assume Mercury to be in hydrostatic equilibrium:

$$
\frac{d P}{d r}=-\rho g
$$

Here, $P$ is pressure, $\rho$ mass density and $g$ gravity. The models also satisfy Poisson's equation

$$
\frac{d g}{d r}=4 \pi \mathrm{G} \rho-2 \frac{g}{r}
$$

where $G$ is the universal gravitational constant. Both equations depend on the density $\rho$, which we calculate from an equation of state (EoS) specifying the dependence of the density on pressure, temperature and composition.

The temperature profile can be calculated by assuming that Mercury is in thermal equilibrium and by specifying at each location the primary method of energy transfer. Thermal equilibrium is the balance between the energy lost by outward energy flux and the internal energy generation and can be expressed as

$$
\frac{d q}{d r}=\rho \epsilon-2 \frac{q}{r}
$$

where $q$ is the outward heat flux per unit area and unit of time and $\epsilon$ the specific heat production rate. If energy is transported mainly by convection, the temperature gradient can be approximated by the adiabatic temperature 
gradient (e.g. Stacey, 1977)

$$
\frac{d T}{d r}=-\frac{\gamma}{K_{S}} \rho g T
$$

where $\gamma=\alpha K_{S} / \rho c_{P}$ is the thermodynamic Grüneisen parameter, $c_{P}$ the specific heat, $\alpha$ the thermal expansion coefficient, and $K_{S}$ the adiabatic bulk modulus. For conduction, the thermal gradient is given by

$$
\frac{d T}{d r}=-\frac{q}{k}
$$

where $k$ is the thermal conductivity.

The temperature is difficult to determine because it depends on the thermal history of Mercury, the unknown amount of radio-active elements in the mantle and crust and the fraction of light elements in the core. Therefore, we here do not solve differential Eqs. (4) and (5) (or (6)) for the thermal quantities $q$ and $T$ in the mantle, but use instead published mantle temperature profiles from studies dedicated to the thermal evolution of Mercury (e.g. Breuer et al., 2007, for a recent review). We consider two temperature profiles, a hot and a cold end-member. Both temperature profiles have been devised under the premise that Mercury is a one-plate planet undergoing stagnant lid convection. As our hot end-member, we select the temperature profile of Buske (2006) with the highest temperature at the core-mantle boundary. At the base of the lithosphere this temperature profile takes about the value of the Earth-mantle solidus proposed by (Zhang and Herzberg, 1994). The temperature profile in Spohn (1991) is taken as our cold end-member. Both temperature profiles have been established for a specific interior structure (e.g. core size), but we use them for all the interior structure models. In practice, the temperature profiles are adapted to a given mantle depth by shortening or lengthening 
their adiabatic part and leaving the other parts unchanged.

The mantle model can then be determined as follows. We integrate Eqs. (2) and (3) from the surface to the core-mantle boundary starting from the boundary conditions at the surface $r=r_{a}$

$$
\begin{aligned}
& P\left(r_{a}\right)=0, \\
& g\left(r_{a}\right)=\frac{G M}{r_{a}^{2}} .
\end{aligned}
$$

These equations and boundary conditions have to be supplemented with equations of state to include pressure-induced compression and thermal expansion effects on the density. The EoS used for the mantle and the crust are discussed in Verhoeven et al. (2005) and Cammarano et al. (2003). In essence, Birch-Murnaghan equations are used and material properties at reference temperature and pressure are corrected to actual mantle temperature and pressure by following first an isobaric and then an isentropic thermodynamic path. The EoS parameters for the minerals of the mantle are given in Table 1.

For the core, we also calculate the temperature profile, given the temperature at the core-mantle boundary. We assume that there are no radio-active elements in the core and that both the liquid outer core and the solid inner core (if present) are convecting and use Eq. (5) for the adiabatic temperature gradient to calculate the temperature in the core. We also discuss the case of a core consisting of an isothermal inner core and a convective outer core. The three governing equations are then given by (2), (3), and (5) for $P, g$, and $T$. At the center, we have the initial condition

$$
g(0)=0
$$


At the core-mantle boundary, the three quantities $P, g$, and $T$ must be continuous.

If an inner core is present, boundary conditions have to be applied at the inner core boundary (ICB) radius $r_{\mathrm{ICB}}$. Besides continuity of $P, g$, and $T$, the temperature at the ICB must be equal to the melting temperature $T_{m}$ of the outer core liquid:

$$
T\left(r_{I C B}\right)=T_{m}\left(x_{S}^{\mathrm{OC}}, P\left(r_{I C B}\right), T\left(r_{I C B}\right)\right)
$$

Here, the dependence of the melting temperature on the composition of the outer core (or sulfur mass concentration $x_{S}^{\mathrm{OC}}$ in the outer core), the pressure and the temperature have been made explicitly.

For a given core size, we integrate 6 differential equations: 3 in the outer core from the core-mantle boundary down and 3 in the solid inner core from the center to the core-mantle boundary. The resulting 6 independent differential equations give rise to 6 integration constants. Since we also solve for the outercore sulfur weight fraction and the location of the boundary between the inner core and the outer core (ICB), we have 8 parameters. These can be determined from 8 conditions: 3 boundary (or continuity) conditions at the CMB, 1 initial condition at the center, 3 continuity conditions at the ICB, and the melting temperature at the ICB.

Once the equations for the structure of Mercury are solved, the density profile in the whole planet is known. The resulting mass and moment of inertia of 
spherical volumes with radius $r$ are then calculated from

$$
\begin{aligned}
m & =4 \pi \int_{0}^{r_{a}} d r \rho(r) r^{2}, \\
I & =\frac{8}{3} \pi \int_{0}^{r_{a}} d r \rho(r) r^{4},
\end{aligned}
$$

and the normalized moment of inertia $M O I$ is

$$
M O I=\frac{I}{m_{a} r_{a}^{2}}
$$

\subsection{The equations of state for the core}

In order to calculate the densities of solid $\gamma-\mathrm{Fe}$ and the liquid $\mathrm{Fe}-\mathrm{S}$ system at local core pressures and temperatures, we use EoS that express the density as a function of pressure and temperature. We correct separately the density for temperature and pressure. At reference pressure, the local density at temperature $T$ of the solid $\gamma-$ Fe is given in terms of the thermal expansion coefficient $\alpha$ as

$$
\rho_{0}(T)=\rho_{\text {ref }} \exp \left[-\int_{T_{\text {ref }}}^{T} d T^{\prime} \alpha\left(T^{\prime}\right)\right] .
$$

Here, $T_{\text {ref }}$ is the reference temperature for the $\gamma-$ Fe. EoS parameters at reference conditions for $\gamma-\mathrm{Fe}$ are given in Table (2). We use a subscript 0 to indicate that the density is calculated at the reference pressure $P_{\text {ref }}: \rho_{0}(T)=$ $\rho\left(T, P_{\text {ref }}\right)$.

We also calculate the elastic moduli at the local temperature $T$. These moduli will be used for the calculation of the response of the models to tidal forcing and the isothermal bulk modulus $K_{T}$ is needed for the calculation of the density at local pressure. The bulk modulus $K_{T}$ and the shear modulus $\mu$ are 
assumed to depend linearly on temperature:

$$
\begin{aligned}
K_{T, 0}(T) & =K_{T, \text { ref }}+\left(\frac{d K_{T}}{d T}\right)_{T_{\text {ref }}}\left(T-T_{\text {ref }}\right), \\
\mu_{0}(T) & =\mu_{\text {ref }}+\left(\frac{d \mu}{d T}\right)_{T_{\text {ref }}}\left(T-T_{\text {ref }}\right) .
\end{aligned}
$$

The values of the EoS parameters for solid $\gamma-$ Fe at reference pressure are given in Table (2). Thermo-elastic properties of liquids can be significantly different from those of their corresponding solid states (Stevenson, 1980). Although the appropriate data relevant to core pressure and temperature conditions is scarce, there currently is a sufficient amount of recent data on the liquid $\mathrm{Fe}-\mathrm{S}$ system for a first-order calculation of the structure of Mercury's core.

We calculate the density of the liquid $\mathrm{Fe}-\mathrm{S}$ mixture from the density of liquid Fe and liquid FeS. As nearly no data is available for densities of $\mathrm{Fe}-\mathrm{S}$ liquids with different fractions of $\mathrm{S}$ relevant for the pressure and temperature conditions in the core of Mercury we approximate the volume of the mixture by the sum of the fractional weighted volume of each component, i.e. we neglect the excessive volume due to mixing. The density of the mixture at local temperature $T$ and reference pressure can then be expressed as

$$
\rho_{0}(T)=\left(\frac{1-x_{\mathrm{FeS}}}{\rho_{0, \mathrm{Fe}}(T)}+\frac{x_{\mathrm{FeS}}}{\rho_{0, \mathrm{FeS}}(T)}\right)^{-1},
$$

where $x_{\mathrm{FeS}}=x_{\mathrm{S}} / 0.3647$ is the weight fraction of liquid FeS. The densities at temperature $T$ of the liquid Fe and the liquid FeS are calculated from Eq. (14) as for the solid $\gamma-\mathrm{Fe}$. The equation of state parameters for liquid $\mathrm{Fe}$ and $\mathrm{FeS}$ relevant for the core model are shown in table 3. The thermal expansivity of the mixture $\alpha_{0}(T)$ at reference pressure is readily computed form the definition

$$
\alpha_{0}(T)=-\left.\frac{\partial \log \rho_{0}(T)}{\partial T}\right|_{P} .
$$


The isothermal bulk modulus of the liquid $\mathrm{Fe}-\mathrm{S}$ system depends on the concentration of sulfur $x_{\mathrm{S}}$. The EoS data for pure Fe (Anderson and Ahrens, 1994), the EoS data for Fe - 0.1wtS (Balog et al., 2003), and the low pressure data from Sanloup et al. (2002) show that the bulk modulus at reference conditions decreases approximatively linearly with increasing $x_{\mathrm{S}}$ for $x_{S}<0.1$. The data of Sanloup et al. (2002) correspond to measurements of $K_{T}$ for Fe - S liquids with different weight fraction of $\mathrm{S}$ in the pressure range $0-5 \mathrm{GPa}$, whereas the $K_{T}$ values of Balog et al. (2003) and Anderson and Ahrens (1994) have been obtained by fitting data measured at different pressures on equations of state. By fitting a linear relation to these data, we obtained

$$
K_{T, \text { ref }}\left(x_{\mathrm{S}}\right)=K_{T, \text { ref }}-K_{x_{\mathrm{S}}} x_{\mathrm{S}}
$$

where $K_{T \text {,ref }}$ is the incompressibility of liquid $\mathrm{Fe}$ and the fitted parameter $K_{x_{\mathrm{S}}}=307 \mathrm{GPa}$.

As is usual for solids, we assume that $K_{T}$ depends linearly on temperature for the liquid mixture:

$$
K_{T, 0}\left(x_{\mathrm{S}}, T\right)=K_{T, \text { ref }}\left(x_{\mathrm{S}}\right)+\left(d K_{T} / d T\right)_{\mathrm{ref}}\left(T-T_{\mathrm{ref}}\right) .
$$

For liquid $\mathrm{Fe}, K_{T}$ is to a very good approximation linear for the temperature range in the core of Mercury. For reasons of lack of data for the derivative of $K_{T}$ with respect to temperature, we use the value for liquid Fe also for liquid $\mathrm{Fe}-\mathrm{S}$ mixtures.

Once the thermoelastic properties of the liquid $\mathrm{Fe}-\mathrm{S}$ mixture and of the solid $\gamma-\mathrm{Fe}$ are calculated at $T$ their values for the compressed matter at local $P$ in the core are determined. For solid and liquid phases, the density and isothermal bulk modulus, and the shear modulus for solid Fe are calculated 
from the 3rd-order Birch-Murnaghan equations (see, e.g. Poirier, 2000):

$$
\begin{aligned}
P= & 3 K_{T, 0} f(1+2 f)^{\frac{5}{2}}\left[1+\frac{3}{2} f\left(K_{T, 0}^{\prime}-4\right)\right. \\
& \left.+\frac{f^{2}}{6}\left(143-63 K_{T, 0}^{\prime}+9\left(K_{T, 0}^{\prime}\right)^{2}+9 K_{T, 0}^{\prime \prime} K_{T, 0}\right)+\ldots\right] \\
K_{T}= & K_{T, 0}(1+2 f)^{\frac{5}{2}}\left\{1-f\left(5-3 K_{T, 0}^{\prime}\right)\right. \\
& \left.+\frac{f^{2}}{2}\left[9 K_{T, 0} K_{T, 0}^{\prime \prime}+\left(3 K_{T, 0}^{\prime}-7\right)\left(3 K_{T, 0}^{\prime}-5\right)\right]\right\} \\
\mu= & \mu_{0}(1+2 f)^{\frac{5}{2}}\left\{1-f\left(5-3 \mu_{0}^{\prime} \frac{K_{T, 0}}{\mu_{0}}\right)\right. \\
& \left.+\frac{f^{2}}{2}\left[9 \mu_{0}^{\prime \prime} \frac{K_{T, 0}^{2}}{\mu_{0}}+9 \mu_{0}^{\prime} \frac{K_{T, 0}}{\mu_{0}}\left(K_{T, 0}^{\prime}-4\right)+35\right]\right\}
\end{aligned}
$$

with

$$
f=\frac{1}{2}\left[\left(\frac{\rho}{\rho_{0}}\right)^{\frac{2}{3}}-1\right] .
$$

In these equations, the right-hand side quantities with subscript 0 have to be evaluated at reference pressure $P_{\text {ref }}$ and local core temperature $T$. The quantities with a superscript / are derivatives with respect to pressure and are, as is usually done when good-quality data are lacking, evaluated at reference conditions (e.g. Sohl and Spohn, 1997). See Table (2) for parameter values for solid $\gamma-\mathrm{Fe}$ and Table (3) for the liquid. For $K_{T}^{\prime}\left(x_{\mathrm{S}}, T_{r e f}\right)$ in the liquid Fe-S system, we use a linear relation derived from fitting data of (Anderson and Ahrens, 1994) for pure Fe and of (Balog et al., 2003) for Fe - 0.1wt\%S. We have

$$
K_{T}^{\prime}\left(x_{\mathrm{S}}\right)=5.8-10 . x_{\mathrm{S}}
$$

The second derivatives (superscript ") of the moduli with respect to pressure are approximated as in Sohl and Spohn (1997):

$$
\begin{aligned}
\mu_{0}^{\prime \prime} & \approx-\frac{35}{9} \frac{\mu_{0}}{K_{T, 0}^{2}}, \\
K_{T, 0}^{\prime \prime} & \approx \frac{1}{K_{T, 0}}\left[K_{T, 0}^{\prime 2}+K_{T, 0}^{\prime}-\frac{143}{9}\right] .
\end{aligned}
$$


For the calculation of the tides of Mercury, we also need the isothermal bulk modulus $K_{T}$ at local core pressure and temperature. It is related to the adiabatic bulk modulus $K_{S}$ through the fundamental thermodynamic relation (Poirier, 2000)

$$
K_{S}(P, T)=K_{T}(P, T)[1+\alpha(P, T) \gamma(P, T) T] .
$$

The thermal expansivity is calculated from

$$
\alpha(P, T) K_{T}(P, T) \approx \alpha\left(P_{r e f}, T\right) K_{T}\left(P_{r e f}, T\right)
$$

This approximate relation is valid whenever the local temperature exceeds the Debye temperature (Poirier, 2000), as in terrestrial planetary cores. The Grüneisen parameter $\gamma$ is computed form the Vashchenko and Zubarev formulation (Anderson, 2000)

$$
\gamma(P, T)=\frac{\frac{1}{2} K_{T}^{\prime}(P, T)-\frac{5}{6}+\frac{2}{9} P K_{T}^{-1}(P, T)}{1-\frac{4}{3} P K_{T}^{-1}(P, T)},
$$

where $K_{T}^{\prime}(P, T)$ is obtained from the Birch-Murnaghan equations as

$$
K_{T}^{\prime}=K_{T, 0}^{\prime}+3 K_{T, 0} K_{T, 0}^{\prime \prime} f
$$

With the adiabatic bulk modulus, shear modulus, and density known at local $(P, T)$ the seismic $\mathrm{P}$ - and $\mathrm{S}$-wave velocities can be calculated from

$$
\begin{aligned}
& V_{p}(P, T)=\sqrt{\frac{K_{S}(P, T)}{\rho(P, T)}}, \\
& V_{s}(P, T)=0,
\end{aligned}
$$


for liquid phases, and

$$
\begin{aligned}
V_{p}(P, T) & =\sqrt{\frac{K_{S}(P, T)+\frac{4}{3} \mu(P, T)}{\rho(P, T)}}, \\
V_{s}(P, T) & =\sqrt{\frac{G(P, T)}{\rho(P, T)}}
\end{aligned}
$$

for solid phases.

\subsection{Results}

In our calculations of the interior structure of Mercury, we choose a radius $r_{\mathrm{CMB}}$ for the core-mantle boundary between $1750 \mathrm{~km}$ and $2200 \mathrm{~km}$ with a step size of $1 \mathrm{~km}$. The lower bound has been chosen to be smaller than the smallest core size possible for a pure iron core, and the upper bound corresponds to a thin silicate shell (mantle and crust) of only $240 \mathrm{~km}$ thick. With our choice of a crust thickness of 100km (Nimmo and Watters, 2004; Grott and Breuer, 2007), the mantle is then only $140 \mathrm{~km}$ deep. Although mass conservation does not exclude the mantle to be thinner (Harder and Schubert, 2001), such a situation seems unlikely. For a given core size, we choose a mantle composition model and a temperature profile for the mantle, a crust density of either $2900 \mathrm{~kg} / \mathrm{m}^{3}$ or $3300 \mathrm{~kg} / \mathrm{m}^{3}$, and then calculate the mantle mineralogy and associated thermodynamic quantities. The mantle model together with the crust determines the boundary conditions for the structure of the core, which we calculate next to obtain a model for the whole interior of Mercury.

Fig. 3 shows the core radius $r_{\mathrm{CMB}}$ as a function of the bulk core sulfur content $x_{\mathrm{S}}$ for 5 different mantle composition models and 2 temperature profiles. The crust density is $2900 \mathrm{~kg} / \mathrm{m}^{3}$ and the inner core temperature profile is adiabatic. 
For any choice of mantle properties, the core radius increases with increasing core sulfur content since more sulfur in the core results in a lower core density. To conserve the mass of Mercury, the core, which is dense compared to the mantle, must then be larger for lower core density. For a similar reason, hot models have a larger core than cold models for a given sulfur concentration in the core: a temperature increase results in a less dense interior and therefore the most dense part of the interior, the core, needs to be larger to conserve the total mass of Mercury. For a mantle temperature increase of $300 \mathrm{~K}$ and for a given core sulfur weight fraction, the core size is about $50 \mathrm{~km}$ or more larger. Note however, that the difference decreases to below about $20 \mathrm{~km}$ for models with pure solid Fe cores, which are the most dense cores.

The core size is between about $1828 \mathrm{~km}$ and $2200 \mathrm{~km}$, which we have chosen as an upper limit. Models with a pure iron core that satisfy the constraints on mass and radius are possible and have the smallest cores (depending on the mantle mineralogy $r_{\mathrm{CMB}}$ is between 1828 and $1871 \mathrm{~km}$ for an adiabatic inner core, if the inner core is conductive the cores are about $10 \mathrm{~km}$ smaller). The models with the largest cores of $2200 \mathrm{~km}$ have a sulfur concentration about $x_{\mathrm{S}}=8.6 \mathrm{wt} \%$ for the hot models and $x_{\mathrm{S}}=7.3 \mathrm{wt} \%$ for the cold models. The mantle mineralogy has a small influence on the core size. At given core sulfur content, the core size difference between different mantle models is at most about $30 \mathrm{~km}$.

Figure 3 also shows the results for the cold FC mantle model with the $3300 \mathrm{~kg} / \mathrm{m}^{3}$ dense crust. The results for the other mantle models with dense crust are not shown here but differ similarly with respect to models with the less dense crust. Globally, models with the denser crust have cores that are about $15 \mathrm{~km}$ smaller than those calculated with the $2900 \mathrm{~kg} / \mathrm{m}^{3}$ dense crust. The models 
with the largest cores of $2200 \mathrm{~km}$ have about $0.6 \mathrm{wt} \%$ more sulfur in the core if the crust is denser.

The core size increase is almost linear in $x_{\mathrm{S}}$. The twist in the curves in Fig. 3 separates models with an inner core from those without. The larger $x_{\mathrm{S}}$ models don't have an inner core for the temperature profiles considered because of the large reducing effect of sulfur on the melting temperature of the core $\mathrm{Fe}-\mathrm{S}$ system. The hot models with an inner core have a small sulfur concentration of less than about 1wt\% (Fig. 4). The cold models have core sulfur concentrations up to almost $5 \mathrm{wt} \%$. For the densest crust of $3300 \mathrm{~km} / \mathrm{m}^{3}$, the sulfur concentration is about $0.2 \mathrm{wt} \%$ higher. This difference decreases with decreasing $x_{\mathrm{S}}$. For a conductive temperature regime in the solid part of the core the results are almost identical (sulfur differences of less than $0.1 \mathrm{wt} \%$ at most) to those of the above adiabatic solid core.

The inner core increases with decreasing sulfur content because less sulfur implies a higher melting temperature (Fig. 4). Therefore, a large pure iron inner core is needed to have a sufficient amount of sulfur in the liquid outer core for the melting temperature to be as low as the actual core temperature corresponding to a chosen mantle temperature profile. In the limit of zero sulfur concentration or a pure iron core, the core is entirely solid for the cold mantle temperature profiles since the CMB temperature of $1850 \mathrm{~K}$ is below the melting temperature for pure iron at the core-mantle boundary pressures of the models with the smallest cores, which is in a range of 1985-2015K (Boehler, 1996; Shen et al., 1998). For the hot mantles with a CMB temperature of $2000 \mathrm{~K}$ and the $2900 \mathrm{~km} / \mathrm{m}^{3}$ dense crust only the FC, MA, and TS mantle models allow for pure solid Fe cores. For the $3300 \mathrm{~km} / \mathrm{m}^{3}$ crust, all mantle models can have solid Fe core. The mantle models that don't allow for a solid 
iron core have lower mantle densities compared to the other models (see Fig. 2 ), and consequently require larger cores to fulfill the global mass constraint. Therefore, they have a lower pressure at the core-mantle boundary and the melting temperature of pure iron at the CMB is lower and below 2000K. For temperatures intermediate between our extreme hot and cold temperature profiles, the relation between inner core size and core bulk sulfur content is intermediate to our results for the two temperature profiles and Mercury models can have small as well as large inner cores for core sulfur concentration between about $1 \mathrm{wt} \%$ and $5 \mathrm{wt} \%$.

The sulfur content in the liquid core, contrary to the global core sulfur concentration, shows little variation with growing inner core due to the strong dependence of the melting temperature of the outer core $\mathrm{Fe}-\mathrm{S}$ system on the sulfur content. Since the temperature profile in the core of our models is determined largely by the chosen mantle temperature profile, models with an inner core that is not zero nor as large as the entire core can only occur in a small range of sulfur concentrations for the outer core. For cold (hot) models, the sulfur concentration in the outer core is about $5 \mathrm{wt} \%$ (1wt\%).

In all our models of Mercury, the outer core has a sulfur content below the eutectic composition, which decreases from about $20 \mathrm{wt} \%$ to about $12 \mathrm{wt} \%$ over a pressure range of $10-40 \mathrm{GPa}$ (Fei et al., 1997, 2000; Stewart et al., 2007). Therefore, compositional buoyancy can occur in the fluid outer core, as would be required for an effective core dynamo to generate a global magnetic field.

Due to the relatively thin mantle the pressure at the core-mantle boundary of our models is between $3 \mathrm{GPa}$ and $9 \mathrm{GPa}$, corresponding to models with a substantial liquid core with sulfur concentration of almost $10 \mathrm{wt} \%$ and to 
models with a solid pure iron core, respectively. At the center of the planet, the pressure ranges from about $30 \mathrm{GPa}$ to about $44 \mathrm{GPa}$. The central temperature of the cold mantle models is about $2300 \mathrm{~K}$ and for the hot models about $2500 \mathrm{~K}$. The difference between the temperature and the melting temperature can be very small in the outer core with typical differences of only a few $10 \mathrm{~K}$ for models with an inner core (Fig. 5). This implies that small changes in temperature can lead to large changes in the size of the inner core. Conductive models have lower temperatures at the center compared to adiabatic models, which show an average temperature increase of about 500K from the coremantle boundary to the center. As a consequence, conductive cores are denser and result in models with somewhat smaller cores (about 12km) due to the global mass constraint (Fig. 6). This small effect decreases with decreasing inner core size.

The average relative mantle density differs by less than $9 \%$ between the various mantle models (Fig. 2). Much larger density differences occur in the liquid core due to differences in the sulfur content. The relative difference in density between the liquid core models with maximal sulfur content and pure iron is about $24 \%$. In the solid inner core, the density differences are much smaller since the composition is pure iron for all models. The density difference can be as large as $1.5 \%$, mainly due to the temperature difference between models, which can be up to a few 100K (see Fig.5). 


\section{Geodesy quantities}

\subsection{Libration}

Because of the Solar gravitational torque on the permanent aspherical figure of Mercury in its elliptical orbit, the rotation of Mercury is not constant and varies periodically about a mean state. The main libration has a period equal to the orbital period of 88 days and its amplitude $\gamma_{1}$ can to a very good approximation be expressed as

$$
\gamma_{1}=\frac{3}{2} \frac{B-A}{C^{m}}\left(1-11 e^{2}+\frac{959}{48} e^{4}+\cdots\right)
$$

(Peale, 1972; Peale et al., 2002; Rambaux et al., 2007), where $A<B$ are the principal equatorial moments of inertia, $C^{m}$ the principal polar moment of inertia of the silicate shell (often this is called the principal moment of inertia of the mantle and we will also use this common practice below), and

$e$ the eccentricity of Mercury. The libration amplitude is proportional to the equatorial moment of inertia difference $B-A$, which can be determined accurately from spacecraft orbiting Mercury, as will be done by MESSENGER and BepiColombo. Therefore, observation of the libration amplitude gives a direct measure of the mantle polar moment of inertia, which is related to the size and density distribution of the mantle. Fig. (7) shows the forced libration amplitude for our interior structure models calculated by assuming the mean moment of inertia difference $B-A=4 \times 10^{-5} M R^{2}$ from the Mariner 10 flybys (Anderson et al., 1987). For the cold mantle models and the $2900 \mathrm{~kg} / \mathrm{m}^{3}$ dense crust, the amplitude is about 21 arcsec for models with a solid core, between 38 arcsec and 56arcsec for models with an inner core, and between 51arcsec and 79arcsec for a fully liquid core. The associated mantle moment of inertia 
relative to the total moment of inertia as a function of the outer core radius is shown in Fig. (8). Since the libration amplitude is inversely proportional to the polar principal moment of inertia of the mantle, it depends mostly on the core radius (Fig. 7) and also increases with decreasing mantle density, in our models by about 5arcsec from the FC to the EC mantle model. The libration amplitude only very weakly depends on the mantle temperature (for given core radius, libration differences between the two temperature profiles $<0.5$ arcsec). Compared to the low density crust, models with the $3300 \mathrm{~kg} / \mathrm{m}^{3}$ dense crust have a larger mantle moment of inertia and a smaller libration amplitude of about 4 arcsec.

Simulations show that the libration of Mercury can be determined with an accuracy of a few arcsec with MESSENGER (Solomon et al., 2001) and maybe even below 1arcsec with BepiColombo (Milani et al., 2001; Pfyffer et al., 2006). Therefore, a precision on the relative mantle moment of inertia of about $2.5 \%$ can be expected. Since this is about 25 times smaller than the total range of relative mantle moment of inertia, libration measurements will strongly constrain the interior of Mercury. As can be seen from Fig. (8), the expected precision on the core size is about $50 \mathrm{~km}$. Since the mantle moment of inertia is mainly determined by the core radius, the observation of the libration amplitude constrains the core composition (see Fig. 3). The expected precision on $x_{\mathrm{S}}$ is smaller than $2 \mathrm{wt} \%$ (Fig. 9). The unknown mantle contributes the most to this error. The core radius estimate is almost independent of the temperature, whereas the estimate of the core sulfur content also depends on the temperature through its effect on the core structure.

Margot et al. (2007) have estimated the libration amplitude by measuring spatial irregularities in the wavefront of radar echoes from Mercury. It is tempting 
to use their value of $35.8 \pm 2$ arcsec to infer the size and composition of the core. However, their results cannot yet be interpreted in terms of the moment of inertia of the mantle since the equatorial moment of inertia difference $B-A$ is currently not known with sufficient precision. A major step forward is expected in the next few years, when the MESSENGER mission will determine the equatorial moment of inertia difference with a precision of about $1 \%$ (Solomon et al., 2001).

If a free libration would be observable, as Earth-based observations by Margot et al. (2007) seem to suggest, the mantle moment of inertia of the mantle could also be determined from the period of this free libration. The free period $\tau_{\text {free }}$ is given by

$$
\tau_{\text {free }}=\frac{2 \pi}{n}\left[\frac{1}{3} \frac{C^{m}}{B-A} \frac{1}{e\left(\frac{7}{2}-\frac{123}{16} e^{2}\right)}\right]^{1 / 2}
$$

(Murray and Dermott, 2000) and is between 8 years and 12 years for our models that don't have fully solid cores (see Fig. 10).

\subsubsection{Obliquity}

Mercury is thought to occupy the Cassini state 1, in which the rotation axis and the orbit normal remain coplanar with the normal to the Laplace plane as both the rotation axis of Mercury and the orbit normal precess about the normal to the Laplace plane with a period of about 280, 000years (Colombo, 1966; Peale, 1969; Ward, 1975). The Laplace plane is defined as the plane about which Mercurys orbit precesses with constant inclination between the two planes. The obliquity, or angle between the rotation axis and the orbit 
normal, of the Cassini state 1 can be expressed as

$$
\epsilon_{\mathrm{C}}=\sin I\left\{\cos I+\frac{n}{\dot{\psi}} \frac{M R^{2}}{C}\left[\frac{J_{2}}{\left(1-e^{2}\right)^{3 / 2}}+2 C_{22}\left(\frac{7}{2} e-\frac{123}{16} e^{3}\right)\right]\right\}^{-1}
$$

(Peale, 1969), where $\epsilon_{C}$ is the obliquity of Cassini state $1, I$ the inclination of the orbital plane to the Laplace plane, and $\dot{\psi}$ the precession rate of Mercury's orbit about the normal to the Laplace plane. Since the polar moment of inertia $C$ is approximately inversely proportional to the obliquity $\epsilon_{C}$ of the Cassini state, the observational determination of the Cassini state obliquity allows determining the polar moment of inertia if all other quantities would be known.

Since the actual obliquity of Mercury may differ from the Cassini state obliquity, an accurate estimate of $C$ can only be obtained when the difference between both obliquities is very small, preferably below the measurement accuracy of the obliquity. Deviations from the Cassini state can be caused by planetary perturbations, by short-periodic variations in the solar torque, and by excitation of free precession. The short-periodic variations in obliquity, similar to the nutations for the Earth and Mars are of the order of 0.1 arcsec or smaller (Carpentier and Roosbeek, 2003; Rambaux and Bois, 2004) and can be neglected. Free precession is thought to be completely damped (Peale, 1974; Ward, 1975) unless a recent $\left(<10^{5}\right.$ years $)$ excitation has occurred, which is very unlikely (Peale, 2005). Moreover, deviations form the Cassini state induced by planetary perturbations have been shown to be small: the spin axis remains within one arcsec of the Cassini state (Peale, 2006). Therefore, the obliquity is expected to be a good approximation of the Cassini obliquity and Eq. (38) can be used to estimate the moment of inertia of Mercury, which is a strong constraint for interior structure models.

Fig. (11) shows that the moment of inertia factor of our Mercury models 
is between about 0.325 and 0.365 , in accordance with the range of values obtained by Harder and Schubert (2001), who however also considered models with much larger core sulfur content up to $36.5 \mathrm{wt} \%$. The obliquity obtained by Margot et al. (2007) of $2.11 \pm 0.1 \mathrm{arcmin}$, can, as for libration, not yet be used because of the large uncertainties in the gravitational coefficients of degree two. However, the space missions MESSENGER and BepiColombo are expected to measure the degree-two gravity coefficients with a precision better than $1 \%$ in the near future. With very accurately known gravity coefficients, an expected $1 \%$ precision on the obliquity (1arcsec precision for a signal of about 100 arcsec) would result in a $1 \%$ precision on the moment of inertia. This would be an order of magnitude improvement with respect to the present theoretical uncertainty on $C$ and would strongly constrain interior structure models of Mercury (see Fig. 11,12). The expected precision on the core size is about $50 \mathrm{~km}$ and the expected precison on $x_{S}$ is about $2 \mathrm{wt} \%$, similarily as for libration. Also as for libration, the error in the core size is mostly due to the unknown mantle density, although the temperature is more important here since the moment of inertia also samples the core directly. For the estimate of the core sulfur content, the temperature is even more important than the mantle mineralogy.

\subsection{Tides}

The gravitational attraction of the Sun causes large tides with amplitudes of the order of $1 \mathrm{~m}$ and main periods at 88days and subharmonics thereof (Van Hoolst and Jacobs, 2003). The tidal acceleration of an unit of matter, or minus the gradient of the tidal potential, is the difference between the local 
acceleration and the orbital acceleration. The degree $l$ part of the frequencydependent tidal potential is conveniently written in the following way

$$
\phi_{l}^{T}(r, \theta, \varphi, \omega)=\left(\frac{r}{r_{a}}\right)^{l} \sum_{m=-l}^{+l} \phi_{l, m}(\theta, \varphi, \omega) .
$$

Beside surface displacements, this periodically varying potential acting on Mercury also causes alterations in Mercury's gravity potential field and gravity variations. Since the reactions of the planet to the forcing are strongly dependent on the interior structure, in particular on the state and size of core, tides are ideally suited for the study of the interior of Mercury. The tidal reactions are customarily described by Love numbers. The radial and tangential displacements are expressed by the numbers $h$ and $l$, and the Love number $k$ describes the external gravity potential variations. For a spherically symmetric, non-rotating, elastic, and isotropic planet, the degree $l$ components of the small tidal surface displacements $\vec{u}_{l}$ can be written as

$$
\begin{aligned}
u_{l, r}\left(r_{a}, \theta, \varphi, \omega\right) & =-\frac{1}{g_{a}} h_{l} \phi_{l, m}(\theta, \varphi, \omega) \\
u_{l, \theta}\left(r_{a}, \theta, \varphi, \omega\right) & =-\frac{1}{g_{a}} l_{l} \frac{\partial \phi_{l, m}(\theta, \varphi, \omega)}{\partial \theta} \\
u_{l, \varphi}\left(r_{a}, \theta, \varphi, \omega\right) & =-\frac{1}{g_{a}} l_{l} \frac{1}{\sin \theta} \frac{\partial \phi_{l, m}(\theta, \varphi, \omega)}{\partial \varphi},
\end{aligned}
$$

where $g_{a}$ is the the surface gravity (e.g. Van Hoolst and Jacobs, 2003). The variations in the external gravitational potential $\Delta \phi$ are due to the tidal potential itself and the potential change resulting from the mass redistribution of the tidally-deformed planet:

$$
\Delta \phi_{l}(r, \theta, \phi, t)=\left[1+k_{l}\left(\frac{r_{a}}{r}\right)^{l+1}\right]\left(\frac{r}{r_{a}}\right)^{l} \phi_{l, m}(\theta, \varphi, \omega) .
$$

The calculations of the deformation and potential variation resulting from the forcing of the tidal potential can be made within the framework of the linear theory of free seismic oscillations extended to forced motions (Dahlen and Tromp, 
1998). The equation of motion for a small unit of matter together with the Poisson equation relating the internal density distribution to the potential are to be solved about the equilibrium configuration. Those equations are further supplemented with the equation of continuity, describing the conservation of mass, and a constitutional relation linking stress to strain. For the elastic setting considered here, the stress-strain relation only depends on the density and on the seismic velocities. By using expansions in spherical harmonics the partial differential equations reduce to a set of eight first-order linear ordinary differential equations. For the calculation of tidal deformations and potential variations only the subset of six differential equations for spheroidal displacements are required.

Compared to the non-forced setting, the only differences are the supplemental tidal force in the equation of motion and an additional term in the surface continuity condition on the radial derivative of the potential. The set of differential equations for tides takes exactly the same form as for free oscillations by replacing the Eulerian perturbation of the gravitational potential $\phi^{E 1}$ by $\Psi=\phi^{E 1}+\phi^{T}$. The non-homogeneous boundary condition on the gravitational potential at the surface can then be expressed as

$$
\frac{d \Psi\left(r_{a}\right)}{d r}+\frac{l+1}{r_{a}} \Psi\left(r_{a}\right)+4 \pi G \rho\left(r_{a}\right) u_{r}\left(r_{a}\right)=\frac{2 l+1}{r_{a}} \phi_{l, m}
$$

The tides of our Mercury models are calculated by numerically integrating the six ordinary differential equations from the center to the surface together with the depth-dependent profiles of density and seismic wave velocities $v_{p}$ and $v_{s}$ (see Figs. 13, 14 and 15). The Love numbers are computed from the solutions at the surface by using the definitions (40), (41) and (43).

The surface displacements and the potential variations have been calculated 
with the tide-generating potential derived by Van Hoolst and Jacobs (2003). The radial displacement at the equator (Fig. 16) has amplitudes between $30 \mathrm{~cm}$ and $1.75 \mathrm{~m}$ and is several times larger than the horizontal displacements. With single-shot laser altimeter measurements, these tidal displacements will be difficult to measure with the MESSENGER and BepiColombo missions to Mercury (Wu et al., 1995; Milani et al., 2001). However, repeated observations with the BepiColombo laser altimeter of the tides, which have a well-known phase and spatial pattern, are expected to lead to tidal amplitudes with a precision of a few percent (at the $1 \sigma$ level) (Koch et al., 2008). The potential variations are 2 orders of magnitude above the expected precision on the degree-two gravity potential determination for the BepiColombo radio science experiment (Milani et al., 2001) but probably difficult to determine by MESSENGER (Solomon et al., 2001). The relative difference between fully solid models and models with large fluid cores can be as high as $60 \%$.

Both Love numbers $h_{2}$ and $k_{2}$ increase with increasing core size (Fig. 17) and increasing sulfur content (Fig. 18). With increasing core size the more easily deformable fluid layer inside the planet facilitates global deformations and therefore leads to larger Love numbers. Fully solid models have significantly smaller Love numbers and radial tidal displacements of about $30 \mathrm{~cm}$.

For a given core size, Love numbers only weakly depend on the mantle temperature and even less on the density of the crust (Fig. 17). The effect of the mantle mineralogy is somewhat larger and the differences between different mantle models amount to almost $10 \%$ between the extreme FC and EC models. This is a consequence of the lower mantle seismic velocities of the EC models. 
With the expected precision of about $1 \%$ on the Love numbers from the future space mission BepiColombo the core radius can be precisely determined with a precision of about $25 \mathrm{~km}$ (Fig. 17) and the sulfur concentration in the core will be estimated with a precision of about 1.5wt\% (Fig. 18). These estimates are better than those from libration and obliquity since tides are more directly sensitive to the core than moments of inertia. However, the estimate on the core sulfur content is only slightly better. The main reason is that the core radius decreases faster with decreasing sulfur content for models with an inner core than for fully liquid core models (see Fig. 3). Therefore, hot models with fully liquid cores with less sulfur can have about equal Love numbers as cold mantles with a solid inner core.

\section{Discussion and Conclusion}

Although only very few data give direct information on Mercury's interior, interior structure models can be developed by assuming a composition for Mercury and by using experimental data on high-pressure and high-temperature properties of mantle and core materials. We have calculated detailed models for the core and for the first time also for the mantle. Because of several unknown basic interior parameters, such as the mantle temperature and the crust density, we have used extreme values to cover the range of plausible values. For other parameters, in particular the material properties at pressure and temperature conditions inside Mercury, we have used values based on the available published data even though they are sometimes uncertain. Because of uncertainties in the modeling, some authors (e.g Harder and Schubert (2001), Hauck et al. (2007)) have used several simplifying assumptions and studied 
global properties of a large number of Mercury models whose model parameters are randomly chosen in a certain interval. Important advantages of studies of individual models as performed here are that more properties can be studied in more detail and that the consequences of changes in model parameters on all model properties can be accurately investigated. In particular, we have studied the relation between the basic core parameters (core radius, inner core radius, and core sulfur content) for five different mantle composition models, two different mantle temperature profiles and two extreme crust density values. We emphasize that these relations are valid within our modeling assumptions and could slightly change when using different material properties and model assumptions. A particular feature of this study is that the effect of temperature on the core is included whereas previous studies either neglected or approximated the temperature profile in the core.

A particular goal of this study was to investigate the effects of the core sulfur content. Our results show that if the temperature at the CMB can be assumed to be above $1850 \mathrm{~K}$, a sulfur content above $10 \mathrm{wt} \%$ would require a silicate shell thinner than $240 \mathrm{~km}$. For a given core radius, denser silicate shells (mantle+crust) are associated with a higher core sulfur content by up to $1 \mathrm{wt} \%$. For a given mantle temperature, the sulfur content of the outer core of models with an inner core is almost constant (within about 1wt\%) irrespective of the bulk core sulfur content or inner core size because the gradient of the melting temperature is nearly equal to the adiabatic temperature gradient in the core. The outer core sulfur content increases with decreasing temperature to about 5wt\% for a CMB temperature of 1850K. Larger sulfur concentrations imply that the core is entirely liquid. However, if Mercury's global magnetic field were generated through compositional convection these fully liquid core 
models would be implausible (for a review, see e.g. Wicht et al., 2007).

For our detailed models of the interior of Mercury, we have calculated the associated libration amplitude, obliquity, tidal deformations, and tidal changes in the external gravitational potential. These geodesy quantity will be accurately determined by future spacecraft missions together with Earth-based radar observations and are considered the main observables to improve our knowledge of Mercury's interior (e.g. Solomon et al., 2001; Milani et al., 2001). The rotation quantities (libration and obliquity) constrain the radial density distribution and the tides are mostly sensitive to the size of the core and to the elastic properties of the core and silicate shell. Since libration is a short-periodic phenomenon, the silicate shell can be considered to librate independently from the core and the libration amplitude is inversely proportional to the moment of inertia of the silicate shell. Because the obliquity reflects a rotation property of the whole planet, it depends on the whole planet moment of inertia. With an expected precision of 1 arcsec on the libration amplitude and obliquity, the moments of inertia can be accurately estimated and therefore insight into the radial density distribution in Mercury can be obtained. For our models with their underlying assumptions, the expected precision on the core radius is about $50 \mathrm{~km}$ and the estimate of the core sulfur content has an error of about $2 \mathrm{wt} \%$. These errors could be largely reduced if the mantle composition were known. Conclusions on the core sulfur content from the determination of the whole planet moment of inertia could be improved by a better estimate of the mantle temperature.

Spacecraft measurements of the tidal surface displacements and the tidal variations in the external gravitational potential give even better estimates of the core size and sulfur content since they are very sensitive to the depth of a 
liquid layer such as the (partially) liquid core. For our models, the core radius can be determined with a precision of about $25 \mathrm{~km}$ if the expected precisions on the Love numbers are about $1 \%$. The expected precision on the core sulfur content is only slightly better than that for the rotation experiments due to the effect of the temperature of the mantle on the core size. It can be concluded that the four geodetic observations will tightly constrain the size and sulfur content of the core of Mercury to a level of precision even better than that currently obtained for Mars. These expected improvements on Mercury's deep interior will have direct consequences for Mercury's formation and will also largely improve scenarios of Mercury's evolution and magnetic history.

\section{Acknowledgments}

This work was financially supported by the Belgian PRODEX program managed by the European Space Agency in collaboration with the Belgian Federal Science Policy Office. The research of AR was supported by the PRODEX program and that of OV by the Action 1 program of the Belgian Federal Science Policy Office. Pierre Vacher is acknowledged for fruitful discussions on the elastic properties of minerals.

\section{References}

Ahrens, T. J., Holland, K. G., Chen, G. Q., Apr. 2002. Phase diagram of iron, revised-core temperatures. Geophys. Res. Lett. 29, 54-1.

Anderson, J. D., Colombo, G., Espsitio, P. B., Lau, E. L., Trager, G. B., Sep. 1987. The mass, gravity field, and ephemeris of Mercury. Icarus 71, 337-349. 
Anderson, O. L., Nov. 2000. The Grüneisen ratio for the last 30years. Geophys. J. Int. 143, 279-294.

Anderson, W. W., Ahrens, T. J., Mar. 1994. An equation of state for liquid iron and implications for the Earth's core. J. Geophys. Res. 99, 4273-4284. Balog, P. S., Secco, R. A., Rubie, D. C., Frost, D. J., Feb. 2003. Equation of state of liquid $\mathrm{Fe}-10 \mathrm{wt} \% \mathrm{~S}$ : Implications for the metallic cores of planetary bodies. J. Geophys. Res. (Solid Earth) 108.

Benz, W., Anic, A., Horner, J., Whitby, J. A., Oct. 2007. The Origin of Mercury. Space Sci. Rev. 132, 189-202.

Benz, W., Slattery, W. L., Cameron, A. G. W., Jun. 1988. Collisional stripping of Mercury's mantle. Icarus 74, 516-528.

Boehler, R., Jul. 1992. Melting of the $\mathrm{Fe}-\mathrm{FeO}$ and the $\mathrm{Fe}-\mathrm{FeS}$ systems at high pressure: Constraints on core temperatures. Earth Planet. Sci. Lett. $111,217-227$.

Boehler, R., Aug. 1996. Fe - FeS eutectic temperatures to 620 kbar. Phys. Earth Planet. Inter. 96, 181-186.

Boynton, W. V., Sprague, A. L., Solomon, S. C., Starr, R. D., Evans, L. G., Feldman, W. C., Trombka, J. I., Rhodes, E. A., Aug. 2007. MESSENGER and the Chemistry of Mercury's Surface. Space Sci. Rev. 131, 85-104.

Breuer, D., Hauck, S. A., Buske, M., Pauer, M., Spohn, T., Oct. 2007. Interior Evolution of Mercury. Space Sci. Rev. 132, 229-260.

Buske, M., 2006. Dreidimensionale thermische Evolutionsmodelle für das Innere von Mars und Merkur. Ph.D. thesis, Georg-August-Universität zu Göttingen.

Cameron, A. G. W., Nov. 1985. The partial volatilization of Mercury. Icarus $64,285-294$.

Cammarano, F., Goes, S., Vacher, P., Giardini, D., Aug. 2003. Inferring upper- 
mantle temperatures from seismic velocities. Phys. Earth Planet. Inter. 138, $197-222$.

Carpentier, G., Roosbeek, F., Jul. 2003. Analytical Development of Rigid Mercury Nutation Series. Celestial Mechanics and Dynamical Astronomy 86, $223-236$.

Colombo, G., Nov. 1966. Cassini's second and third laws. Astronomical Journal $71,891-896$.

Dahlen, F. A., Tromp, J., September 1998. Theoretical Global Seismology. Princeton University Press.

Fegley, B., Cameron, A. G. W., Apr. 1987. A vaporization model for iron/silicate fractionation in the Mercury protoplanet. Earth Planet. Sci. Lett. 82, 207-222.

Fei, Y., 1995. Mineral Physics and Crystallography: A Handbook of Physical Constants. Vol. 2 of AGU Reference Shelf 2. AGU, Washington, D.C., Ch. Thermal expansion, pp. 29-44.

Fei, Y., Bertka, C. M., Finger, L. W., March 1997. High-Pressure Iron-Sulfur Compound, $\mathrm{Fe}_{3} \mathrm{~S}_{2}$, and Melting Relations in the Fe - FeS System. Science $275,1621-1623$.

Fei, Y., Li, J., Bertka, C. M., Prewitt, C. T., 2000. Structure type and bulk modulus of $\mathrm{Fe}_{3} \mathrm{~S}$, a new iron-sulfur compound. American Mineralogist 85 (11-12), 1830-1833.

Fei, Y., Prewitt, C. T., Mao, H.-K., Bertka, C. M., Jun. 1995. Structure and Density of FeS at High Pressure and High Temperature and the Internal Structure of Mars. Science 268, 1892-1894.

Goettel, K. A., 1988. Present bounds on the bulk composition of Mercury - Implications for planetary formation processes. Mercury, University of Arizona Press, pp. 613-621. 
Grott, M., Breuer, D., 2007. Viscous topographic relaxation on Mercury revisited: Improved constraints on Mercury's Crustal Thickness. Icarus.

Harder, H., Schubert, G., May 2001. Sulfur in Mercury's Core? Icarus 151, $118-122$.

Hauck, S. A., Aurnou, J. M., Dombard, A. J., Sep. 2006. Sulfur's impact on core evolution and magnetic field generation on Ganymede. J. Geophys. Res. (Planets) 111, 1-14.

Hauck, S. A., Dombard, A. J., Phillips, R. J., Solomon, S. C., Jun. 2004. Internal and tectonic evolution of Mercury. Earth Planet. Sci. Lett. 222, $713-728$.

Hauck, S. A., Solomon, S. C., Smither, A., July 2007. Predicted recovery of Mercury's internal structure by MESSENGER. Geophys. Res. Lett. 34.

Jeanloz, R., Mitchell, D. L., Sprague, A. L., de Pater, I., Jun. 1995. Evidence for a basalt-free surface on Mercury and implications for internal heat. Science 268, 1455-1457.

Kaiura, G. H., Toguri, J. M., 1979. Densities of the Molten FeS, FeS - $\mathrm{Cu}_{2} \mathrm{~S}$ and $\mathrm{Fe}-\mathrm{S}-\mathrm{O}$ Systems Utilizing a Bottom-Balance Archimedean Technique. Canadian Metallurgical Quarterly 18 (2), 155-164.

Koch, C., Christensen, U., Kallenbach, R., 2008. Simultaneous determination of global topography, tidal Love number and libration amplitude of Mercury by laser altimetry. Planet. Space Sci. 56 (9), 1226-1237.

Krot, A. N., Meibom, A., Russell, S. S., O’D. Alexander, C. M., Jeffries, T. E., Keil, K., Mar. 2001. A New Astrophysical Setting for Chondrule Formation. Science 291, 1776-1779.

Kuwayama, Y., Hirose, K., 2004. Phase relations in the system Fe - FeSi at 21 GPa. American Mineralogist 89 (2-3), 273-276.

Lewis, J. S., 1988. Origin and composition of Mercury. Mercury, University of 
Arizona Press, pp. 651-666.

Li, J., Fei, Y., Mao, H. K., Hirose, K., Shieh, S. R., Dec. 2001. Sulfur in the Earth's inner core. Earth Planet. Sci. Lett. 193, 509-514.

Margot, J. L., Peale, S. J., Jurgens, R. F., Slade, M. A., Holin, I. V., 2007. Large Longitude Libration of Mercury Reveals a Molten Core. Science 316 (5825), 710-714.

Matsui, M.and Parker, S. C., Leslie, M., 2000. The MD simulation of the equation of state of $\mathrm{MgO}$ : as a pressure calibration standard at high temperature and high pressure. American Mineralogist 85, 312-316.

Milani, A., Rossi, A., Vokrouhlický, D., Villani, D., Bonanno, C., Dec. 2001. Gravity field and rotation state of Mercury from the BepiColombo Radio Science Experiments. Planet. Space Sci. 49, 1579-1596.

Moore, P. M., Araki, T., 1972. Atomic arrangement of merwinite, $\mathrm{Ca}_{3} \mathrm{Mg}\left[\mathrm{SiO}_{4}\right]_{2}$, an unusual dense-packed, structure of geophysical interest. American Mineralogist 57, 1355-1374.

Morgan, J. W., Anders, E., Dec. 1980. Chemical Composition of Earth, Venus, and Mercury. Proceedings of the National Academy of Science 77, 69736977.

Murray, C. D., Dermott, S. F., 2000. Solar system dynamics. Cambridge University Press.

Nimmo, F., Watters, T. R., Jan. 2004. Depth of faulting on Mercury: Implications for heat flux and crustal and effective elastic thickness. Geophys. Res. Lett. 31.

Peale, S. J., Apr. 1969. Generalized Cassini's Laws. Astronomical Journal 74, $483-489$.

Peale, S. J., Aug. 1972. Determination of Parameters Related to the Interior of Mercury. Icarus 17, 168-173. 
Peale, S. J., Jun. 1974. Possible histories of the obliquity of Mercury. Astronomical Journal 79, 722-744.

Peale, S. J., Nov. 2005. The free precession and libration of Mercury. Icarus $178,4-18$.

Peale, S. J., Apr. 2006. The proximity of Mercury's spin to Cassini state 1 from adiabatic invariance. Icarus 181, 338-347.

Peale, S. J., Phillips, R. J., Solomon, S. C., Smith, D. E., Zuber, M. T., Sep. 2002. A procedure for determining the nature of Mercury's core. Meteoritics and Planetary Science 37, 1269-1283.

Pfyffer, G., Rambaux, N., Rivoldini, A., van Hoolst, T., Dehant, V., 2006. Determination of libration amplitudes from orbit. In: European Planetary Science Congress 2006. p. 568.

Poirier, J.-P., Mar. 2000. Introduction to the Physics of the Earth's Interior. Introduction to the Physics of the Earth's Interior, by Jean-Paul Poirier, pp. 326. ISBN 052166313X. Cambridge, UK: Cambridge University Press, March 2000.

Rambaux, N., Bois, E., Jan. 2004. Theory of the Mercury's spin-orbit motion and analysis of its main librations. Astronomy and Astrophysic 413, 381393.

Rambaux, N., van Hoolst, T., Dehant, V., Bois, E., Jun. 2007. Inertial coremantle coupling and libration of Mercury. Astronomy and Astrophysics 468, $711-719$.

Robinson, M. S., Taylor, G. J., Jun. 2001. Ferrous oxide in Mercury's crust and mantle. Meteoritics and Planetary Science 36, 841-847.

Rubie, D. C., Gessmann, C. K., Frost, D. J., May 2004. Partitioning of oxygen during core formation on the Earth and Mars. Nature 429, 58-61.

Sanloup, C., Fei, Y., Oct. 2004. Closure of the Fe - S - Si liquid miscibility 
gap at high pressure. Phys. Earth Planet. Inter. 147, 57-65.

Sanloup, C., Guyot, F., Gillet, P., Fei, Y., Nov. 2002. Physical properties of liquid Fe alloys at high pressure and their bearings on the nature of metallic planetary cores. J. Geophys. Res. (Solid Earth) 107, 4-1.

Saxena, S. K., Shen, G., Dec. 1992. Assessed data on heat capacity, thermal expansion, and compressibility for some oxides and silicates. J. Geophys. Res. 97, 19813-19825.

Schubert, G., Ross, M. N., Stevenson, D. J., Spohn, T., 1988. Mercury's thermal history and the generation of its magnetic field. Mercury, University of Arizona Press, pp. 429-460.

Shen, G., Mao, H.-k., Hemley, R. J., Duffy, T. S., Rivers, M. L., 1998. Melting and crystal structure of iron at high pressures and temperatures. Geophys. Res. Lett. 25, 373-376.

Siegfried, II, R. W., Solomon, S. C., Oct. 1974. Mercury - Internal structure and thermal evolution. Icarus 23, 192-205.

Smyth, J. R., McCormick, T. C., 1995. Mineral Physics and Crystallography: A Handbook of Physical Constants. Vol. 2 of AGU Reference Shelf 2. AGU, Washington, DC, Ch. Crystallographic data for minerals, pp. 1-17.

Sobolev, S. V., Babeyko, A. Y., Sep. 1994. Modeling of mineralogical composition, density and elastic wave velocities in anhydrous magmatic rocks. Surveys in Geophysics 15, 515-544.

Sohl, F., Spohn, T., Jan. 1997. The interior structure of Mars: Implications from SNC meteorites. J. Geophys. Res. 102, 1613-1636.

Solomon, S. C., McNutt, R. L., Gold, R. E., Acuña, M. H., Baker, D. N., Boynton, W. V., Chapman, C. R., Cheng, A. F., Gloeckler, G., Head, III, J. W., Krimigis, S. M., McClintock, W. E., Murchie, S. L., Peale, S. J., Phillips, R. J., Robinson, M. S., Slavin, J. A., Smith, D. E., Strom, R. G., 
Trombka, J. I., Zuber, M. T., Dec. 2001. The MESSENGER mission to Mercury: scientific objectives and implementation. Planet. Space Sci. 49, $1445-1465$.

Spohn, T., Apr. 1991. Mantle differentiation and thermal evolution of Mars, Mercury, and Venus. Icarus 90, 222-236.

Spohn, T., Sohl, F., Wieczerkowski, K., Conzelmann, V., Dec. 2001. The interior structure of Mercury: what we know, what we expect from BepiColombo. Planet. Space Sci. 49, 1561-1570.

Sprague, A. L., Kozlowski, R. W. H., Witteborn, F. C., Cruikshank, D. P., Wooden, D. H., May 1994. Mercury: Evidence for anorthosite and basalt from mid-infrared (7.3-13.5 micrometers) spectroscopy. Icarus 109, 156-167.

Stacey, F. D., 1977. Applications of thermodynamics to fundamental earth physics. Geophysical Surveys 3, 175-204.

Stevenson, D. J., Apr. 1980. Applications of liquid state physics to the earth's core. Phys. Earth Planet. Inter. 22, 42-52.

Stevenson, D. J., Spohn, T., Schubert, G., Jun. 1983. Magnetism and thermal evolution of the terrestrial planets. Icarus 54, 466-489.

Stewart, A. J., Schmidt, M. W., van Westrenen, W., Liebske, C., Jun. 2007. Mars: A New Core-Crystallization Regime. Science 316, 1323-.

Taylor, G. I., Scott, E. R. F., 2005. Mercury. In: Davis, A. M. (Ed.), Treatise on Geochemistry, Meteorites, Comets and Planets. Vol. 1. Elsevier, Amsterdam, pp. 477-485.

Tsuno, K., Ohtani, E., Terasaki, H., Jan. 2007. Immiscible two-liquid regions in the $\mathrm{Fe}-\mathrm{O}-\mathrm{S}$ system at high pressure: Implications for planetary cores. Phys. Earth Planet. Inter. 160, 75-85.

Vacher, P., Mocquet, A., Sotin, C., Apr. 1998. Computation of seismic profiles from mineral physics: the importance of the non-olivine components for 
explaining the $660 \mathrm{~km}$ depth discontinuity. Phys. Earth Planet. Inter. 106, $275-298$.

Van Hoolst, T., Jacobs, C., Nov. 2003. Mercury's tides and interior structure. J. Geophys. Res. (Planets) 108.

Van Hoolst, T., Sohl, F.and Holin, I., Verhoeven, O., Dehant, V., Spohn, T., 2007. Mercury's Interior Structure, Rotation, and Tides. Space Sci. Rev. $132(2-4), 203-227$.

Verhoeven, O., Rivoldini, A., Vacher, P., Mocquet, A., Choblet, G., Menvielle, M., Dehant, V., Van Hoolst, T., Sleewaegen, J., Barriot, J.-P., Lognonné, P., Apr. 2005. Interior structure of terrestrial planets: Modeling Mars' mantle and its electromagnetic, geodetic, and seismic properties. J. Geophys. Res. (Planets) 110 (E9).

Verhoeven, O., Tarits, P., Rivoldini, A., Van Hoolst, T., 2008. Electrical conductivity of Mercury: implication on formation and evolution. Planet. Space Sci.

Ward, W. R., Jan. 1975. Tidal friction and generalized Cassini's laws in the solar system. Astronomical Journal 80, 64-70.

Wasson, J. T., 1988. The building stones of the planets. Mercury, University of Arizona Press, pp. 622-650.

Weidenschilling, S. J., Jul. 1978. Iron/silicate fractionation and the origin of Mercury. Icarus 35, 99-111.

Wetherill, G. W., 1988. Accumulation of Mercury from planetesimals. Mercury, University of Arizona Press, pp. 670-691.

Wicht, J., Mandea, M., Takahashi, F., Christensen, U. R., Matsushima, M., Langlais, B., Oct. 2007. The Origin of Mercury's Internal Magnetic Field. Space Sci. Rev. 132, 261-290.

Wu, X., Bender, P. L., Rosborough, G. W., Jan. 1995. Probing the interior 
structure of Mercury from an orbiter plus single lander. J. Geophys. Res. $100,1515-1525$.

Zhang, J., Herzberg, C., Sep. 1994. Melting experiments on anhydrous peridotite KLB-1 from 5.0 to 22.5 GPa. J. Geophys. Res. 99, 17729-17742.

Zhang, L., Fei, Y., 2008. Effect of $\mathrm{Ni}$ on $\mathrm{Fe}-\mathrm{FeS}$ phase relations at high pressure and high temperature. Earth Planet. Sci. Lett. 268 (1-2), 212-218. Zuber, M. T., Aharonson, O., Aurnou, J. M., Cheng, A. F., Hauck, S. A., Heimpel, M. H., Neumann, G. A., Peale, S. J., Phillips, R. J., Smith, D. E., Solomon, S. C., Stanley, S., Aug. 2007. The Geophysics of Mercury: Current Status and Anticipated Insights from the MESSENGER Mission. Space Sci. Rev. 131, 105-132. 
TABLES

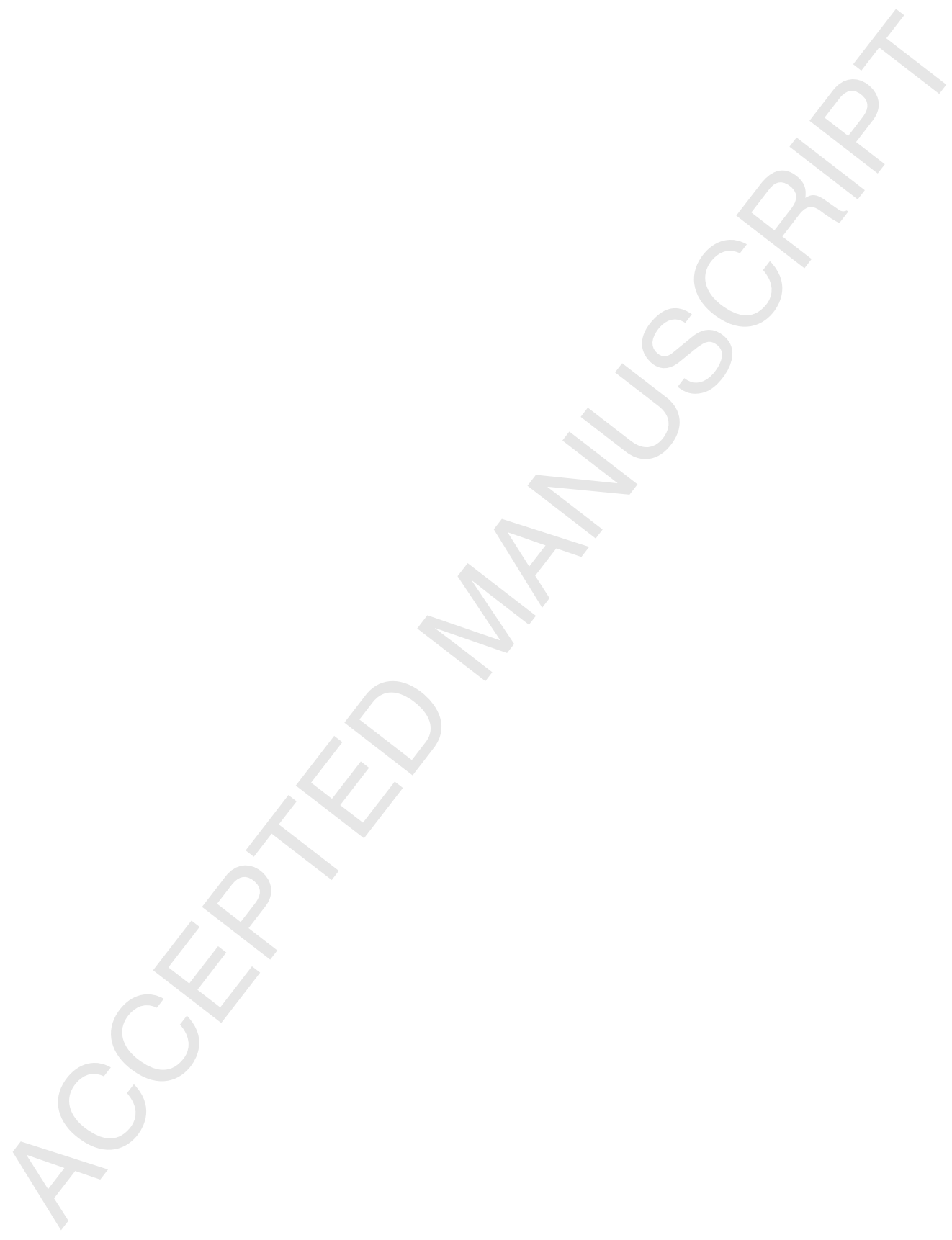




\begin{tabular}{|c|c|c|c|c|c|c|c|c|c|c|c|c|c|}
\hline & $\begin{array}{c}\rho_{0} \\
10^{3} \mathrm{~kg} / \mathrm{m}^{3}\end{array}$ & $\begin{array}{c}\rho_{0, F e} \\
10^{3} \mathrm{~kg} / \mathrm{m}^{3}\end{array}$ & $\begin{array}{c}a_{0} \\
1 /\left(10^{5} \mathrm{~K}\right)\end{array}$ & $\begin{array}{c}b_{0} \\
1 /\left(10^{8} \mathrm{~K}^{2}\right)\end{array}$ & $\begin{array}{l}c_{0} \\
\mathrm{~K}\end{array}$ & $\begin{array}{c}d_{0} \\
10^{-2}\end{array}$ & $\gamma_{t h_{0}}$ & $\begin{array}{l}K_{S, 0} \\
\mathrm{GPa}\end{array}$ & $\begin{array}{c}K_{S, 0, F e} \\
\mathrm{GPa}\end{array}$ & $\left.\frac{\partial K_{S}}{\partial P}\right|_{0}$ & $\begin{array}{l}\left.\frac{\partial K_{S}}{\partial T}\right|_{0} \\
\mathrm{GPa} / \mathrm{K}\end{array}$ & $\begin{array}{c}G_{0} \\
\mathrm{GPa}\end{array}$ & $\begin{array}{c}G_{0, F e} \\
\mathrm{GPa}\end{array}$ \\
\hline ol & 3.222 & 1.182 & 2.832 & 0.758 & 0. & 0. & 1.14 & 129. & 0. & 4.2 & -0.016 & 81 & -31 \\
\hline opx & 3.194 & 0.8 & 2.86 & 0.72 & 0. & 0. & 1.05 & 109. & -5 . & 7.0 & -0.012 & 75 & 10 \\
\hline Ca-px & 3.277 & 0.38 & 2.32 & 1.88 & 0. & 0. & 1.06 & 105. & 13. & $6.2-1.9 x_{F e}$ & -0.013 & 67. & -6 . \\
\hline gt & 3.565 & 0.76 & 2.08 & 1.43 & 0. & 0. & 1.17 & 171 & 15. & 4.4 & -0.021 & 92. & 7. \\
\hline an & 2.75 & - & 2.34 & 1.21 & 2.19 & 0.52 & 1 & 88.9 & - & 6.6 & -0.011 & 40.4 & - \\
\hline $\mathrm{sp}$ & 3.58 & 0.7 & 6.97 & -0.11 & -5.04 & -3.08 & 1.21 & 196.7 & 13.6 & 4.9 & -0.016 & 108. & -24 \\
\hline ky & 3.68 & - & 5.4 & -0.02 & -1.55 & -1.5 & 1.17 & 191.3 & & 4. & -0.048 & 96.6 & - \\
\hline coe & 2.911 & - & 2.47 & 0.29 & -0.13 & -0.97 & 1 & 98. & - & 4.3 & -0.015 & 61.7 & - \\
\hline cor & 3.988 & - & 4.2 & -0.08 & -1.06 & -1.14 & 1 & 257. & - & 4.4 & -0.014 & 162. & - \\
\hline$q$ & 2.65 & - & 6. & - & - & - & 1 & 37.7 & - & 6.4 & -0.009 & 44.5 & - \\
\hline per & 3.585 & - & 3.64 & 0.835 & 0.95 & 0.085 & 1.47 & 162 & - & 4.26 & -0.028 & 132 & - \\
\hline merw & 3.33 & - & 2.52 & 1.53 & 0. & 0 & 1 & 129. & - & 4.2 & -0.016 & 81 & - \\
\hline
\end{tabular}

Table 1

Elastic properties of minerals at STP conditions $\left(P_{0}=10^{5} \mathrm{~Pa}, T_{0}=298 \mathrm{~K}\right)$. Data are taken from the compilation of Saxena and Shen (1992), Sobolev and Babeyko (1994), Vacher et al. (1998), Cammarano et al. (2003), Verhoeven et al. (2005). Density and elastic properties of periclase are from Smyth and McCormick (1995) and Matsui and Leslie (2000) and references therein. Density and thermal expansion for merwinite are from Moore and Araki (1972) and from Fei (1995), respectively. Values in italics are assumed. Elastic properties of merwinite assumed identical to olivine. $\rho_{0}, K_{S, 0}$ and $G_{0}$ refer to density, bulk and shear modulus, respectively. $\left.\frac{\partial K_{S}}{\partial P}\right|_{0}$ and $\left.\frac{\partial K_{S}}{\partial T}\right|_{0}$ are the derivatives of the bulk modulus with respect to $P$ and $T$, respectively. $\left.\frac{\partial G^{(i)}}{\partial P}\right|_{0}$ and $\left.\frac{\partial G^{(i)}}{\partial T}\right|_{0}$ are the derivatives of the shear modulus with respect to $P$ and $T$, respectively. $\rho_{0, F e}, K_{S, 0, F e}$ and $G_{0, F e}$ give the iron content dependence of the density, bulk and shear modulus, respectively. The thermal expansion is: $\alpha\left(P_{0}, T\right)=a_{0}+b_{0} T-c_{0} T^{-2}+d_{0} T^{-1}$ where all $c_{0}$ and $d_{0}$ are equal to zero except for the coesite for which $c_{0}=-0.13$ and $d_{0}=0.9710^{-2}$ (Saxena and Shen, 1992). $\gamma_{t h, 0}$ denotes the Grüneisen parameter. Values in italics are assumed to be equal to 1. Abbreviations: an: anorthite, cpx: Ca-pyroxene, coe: coesite, cor: corundum, gt: garnet, ky: kyanite, merw: merwinite, ol: olivine, opx: orthopyroxene, per: periclase, q: quartz, sp: spinel, LP: low p̧̊essure, HP: high pressure. 


\begin{tabular}{|c|c|}
\hline$P_{\text {ref }}$ & $10^{5} \mathrm{~Pa}$ \\
\hline$T_{\text {ref }}$ & $1573 \mathrm{~K}$ \\
\hline$\rho_{\text {ref }}$ & $7413 \mathrm{~kg} / \mathrm{m}^{-3}$ \\
\hline$K_{T, \text { ref }}$ & $103 \mathrm{GPa}$ \\
\hline$K_{T, \text { ref }}^{\prime}$ & 5 \\
\hline$\left(d K_{T} / d T\right)_{\mathrm{ref}}$ & $-0.02 \mathrm{GPa} / \mathrm{K}$ \\
\hline$\alpha_{0}(T)$ & $7.710^{-5} 1 / \mathrm{K}$ \\
\hline$\mu_{\text {ref }}$ & $62.6 \mathrm{G}$ \\
\hline$\mu_{\text {ref }}^{\prime}$ & 2.7 \\
\hline$(d \mu / d T)_{\mathrm{ref}}$ & $-0.03 \mathrm{GPa} / \mathrm{K}$ \\
\hline
\end{tabular}

Table 2

Equation of state parameter for $\gamma-$ Fe from Ahrens et al. (2002).

FIGURE CAPTIONS 


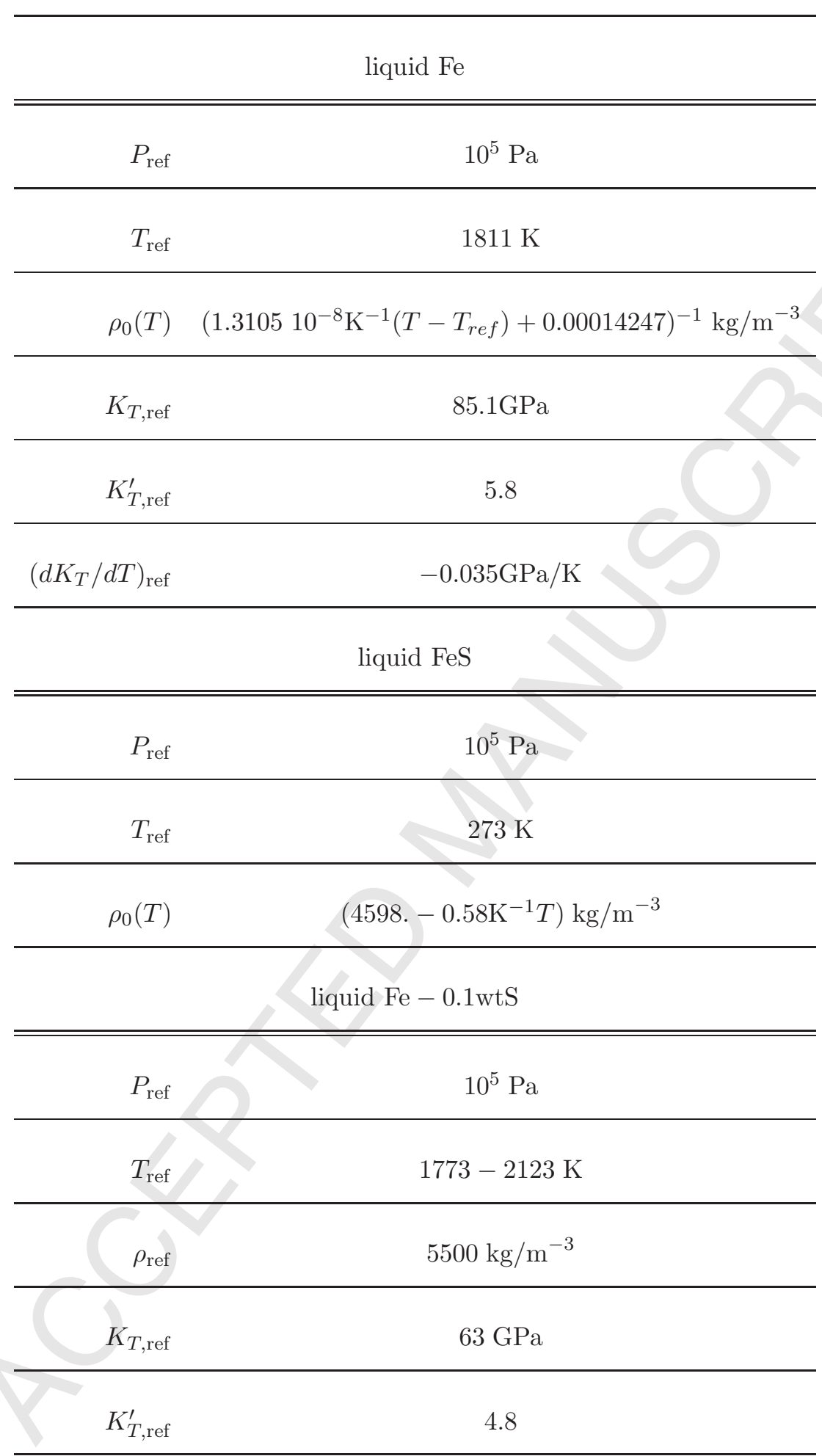

Table $\overline{3}$

Equation of state parameter for liquid Fe from Anderson and Ahrens (1994), liquid FeS Kaiura and Toguri (1979), and liquid Fe - 0.1wtS Balog et al. (2003). 


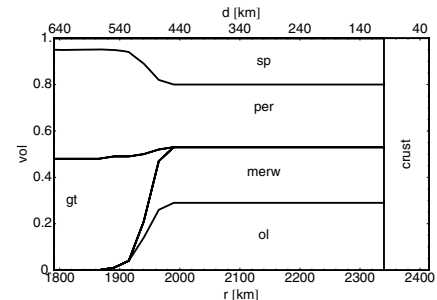

a. FC, cold

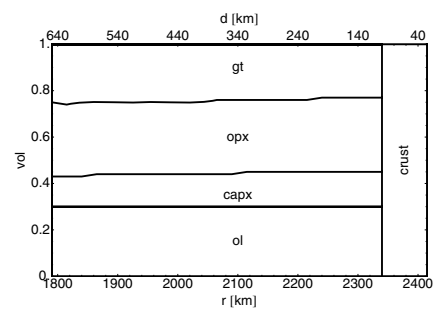

c. MA, cold

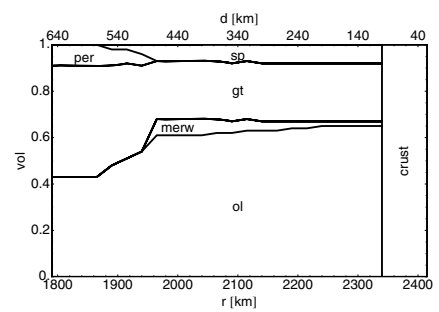

e. TS, cold

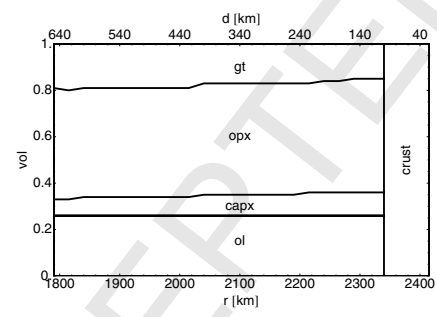

g. MC, cold

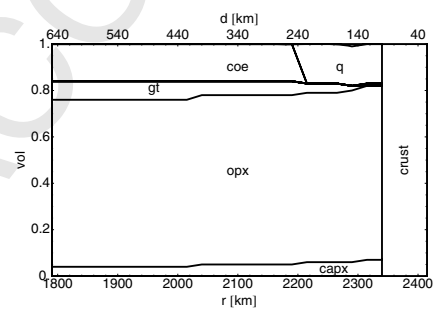

i. EC, cold

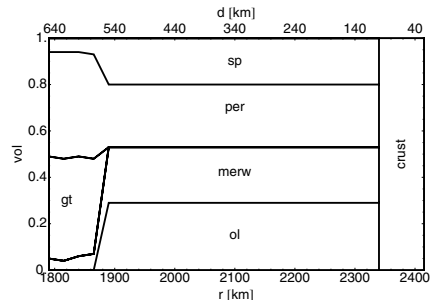

b. FC, hot

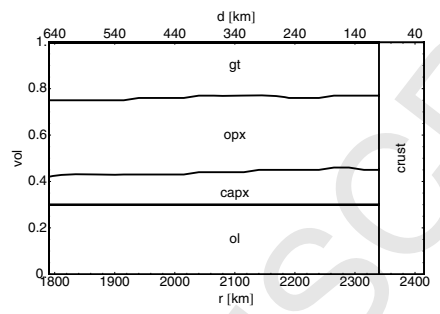

d. $M A$, hot

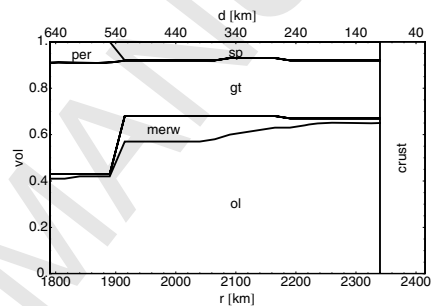

f. TS, hot

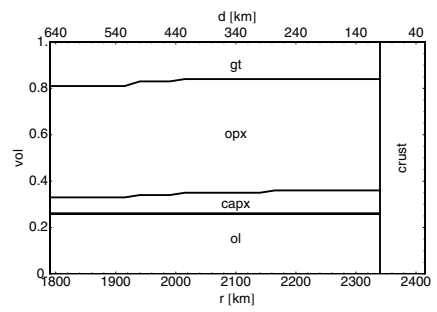

h. $M C$, hot

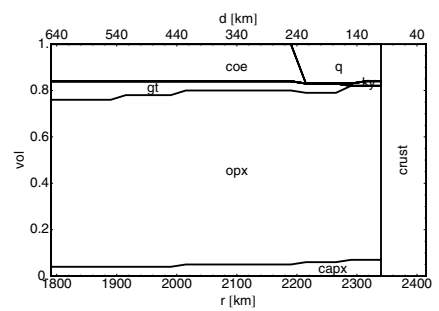

j. EC, hot

Fig. 1. Depth dependent volume fraction of the five mineralogy models. (an: anorthite, cpx: Ca-pyroxene, coe: coesite, cor: corundum, gt: garnet, 55 : kyanite, merw: merwinite, ol: olivine, opx: orthopyroxene, per: periclase, q: quartz, sp: spinel, LP: low pressure, HP: high pressure) 


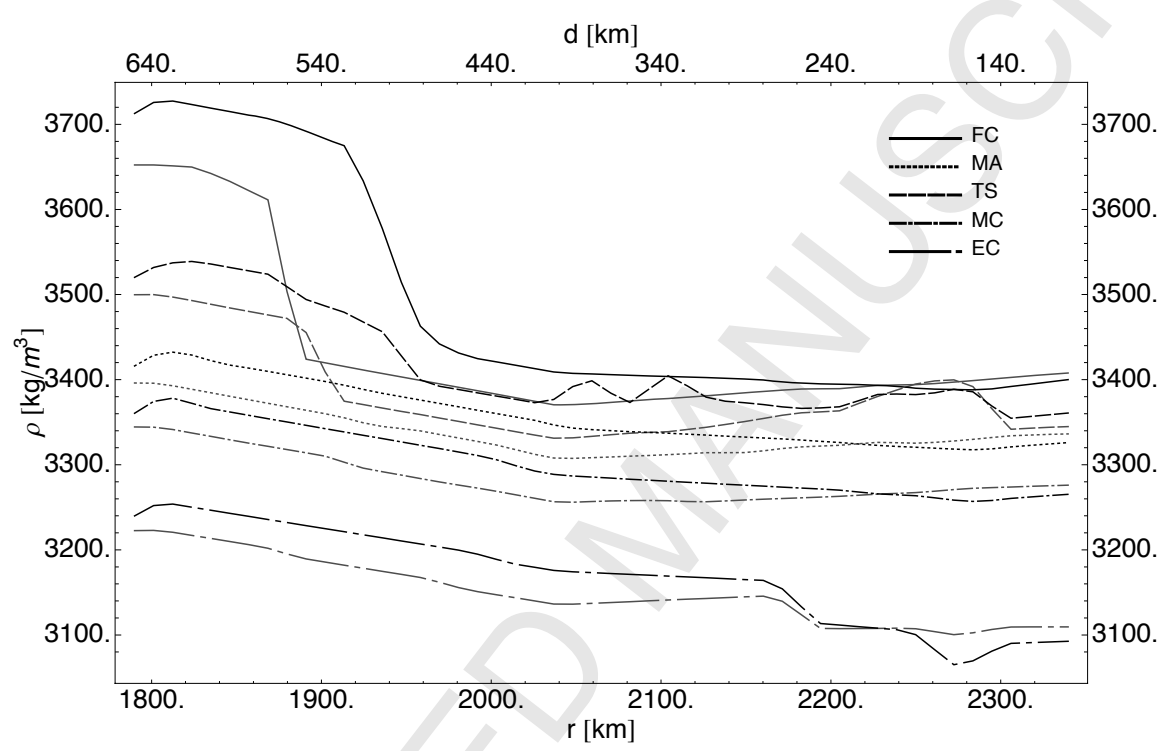

Fig. 2. Density profiles associated to the five mantle mineralogy models. Hot mantle models are depicted in gray and cold models in black. 


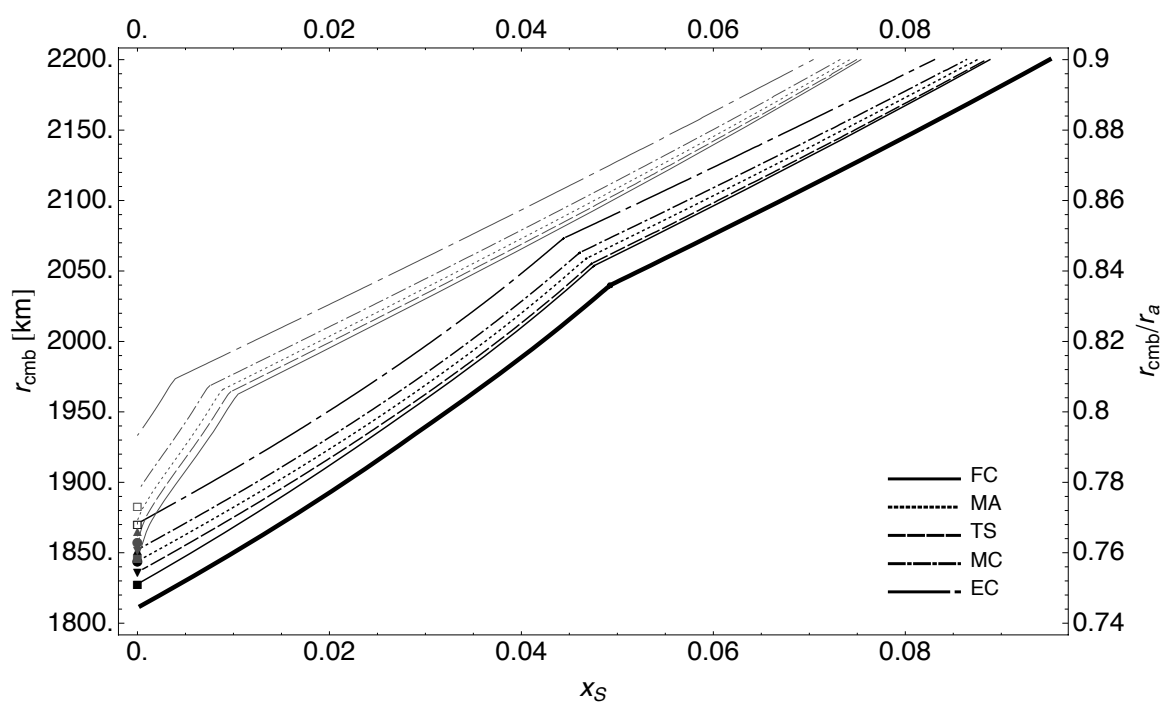

Fig. 3. Core size as a function of core sulfur weight fraction for hot (gray) and cold (black) models. Crust density is $2900 \mathrm{~kg} / \mathrm{m}^{3}$ except for the results for the cold FC model, which are also shown for the crust density of $3300 \mathrm{~kg} / \mathrm{m}^{3}$ (thick black). 


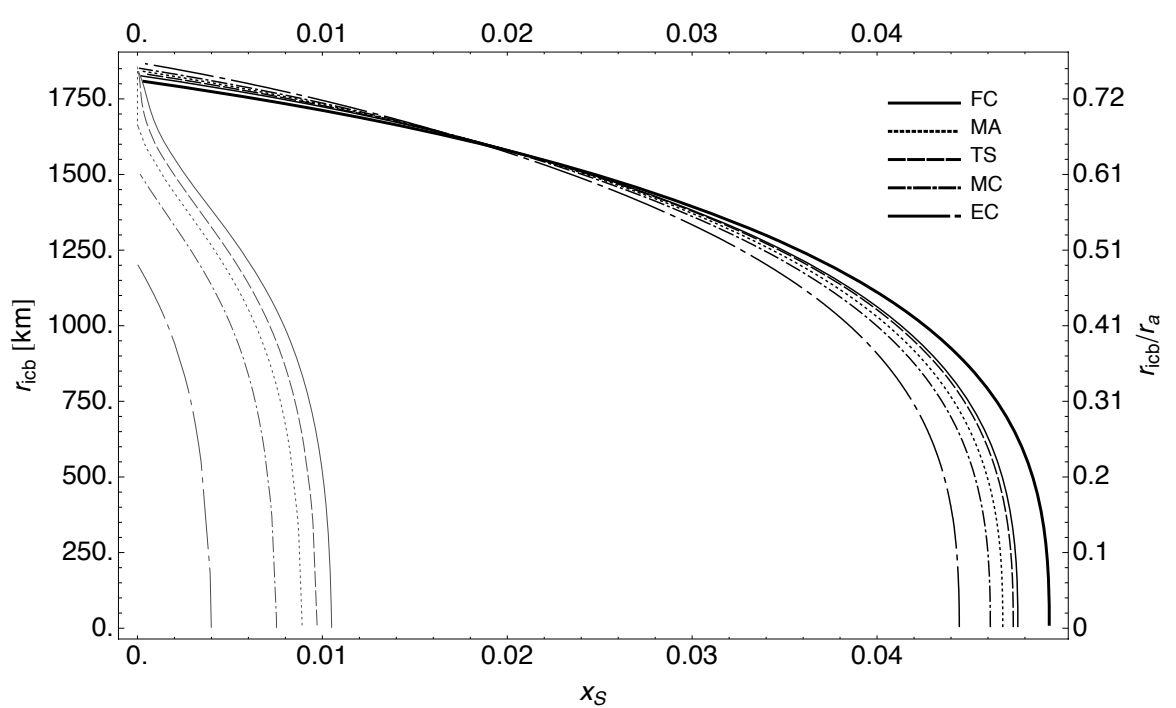

Fig. 4. Inner core size as a function of core sulfur weight fraction for hot (gray) and cold (black) models. Crust density is $2900 \mathrm{~kg} / \mathrm{m}^{3}$ except for the results for the cold FC model, which are also shown for the crust density of $3300 \mathrm{~kg} / \mathrm{m}^{3}$ (thick black). 


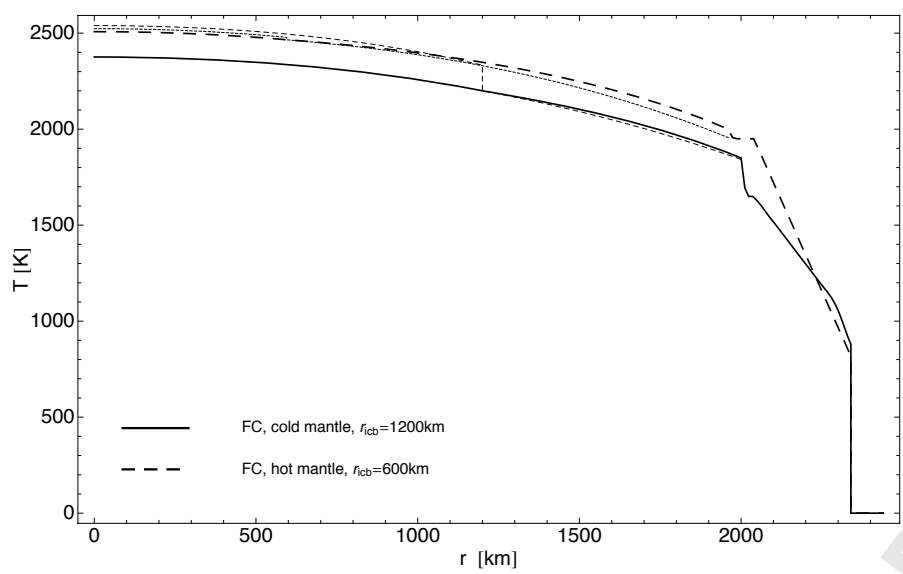

Fig. 5. Temperature profiles for hot (dashed) and cold (continuous) mantle temperatures. The light dashed curves are the melting temperatures.

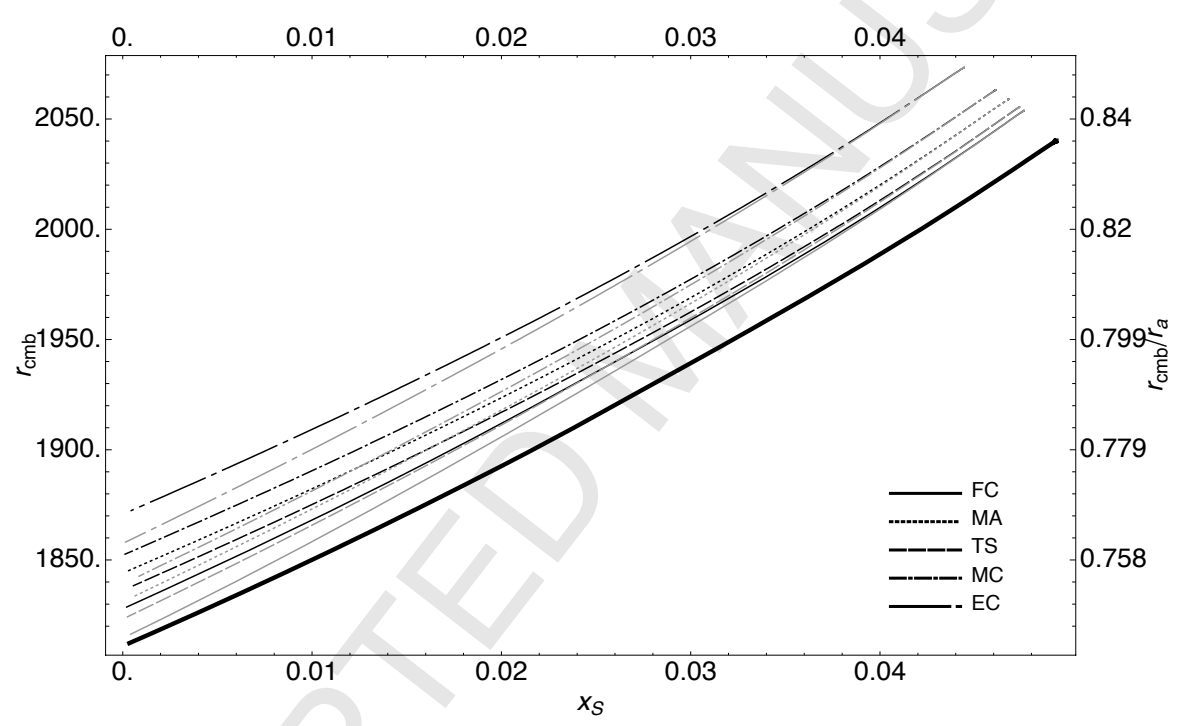

Fig. 6. Core radius as a function of core sulfur weight fraction for models with inner cores and cold mantle temperature for adiabatic (gray) and conductive (black) inner core temperature profiles. Crust density is $2900 \mathrm{~kg} / \mathrm{m}^{3}$ except for the results for the cold FC model, which are also shown for the crust density of $3300 \mathrm{~kg} / \mathrm{m}^{3}$ (thick black). 


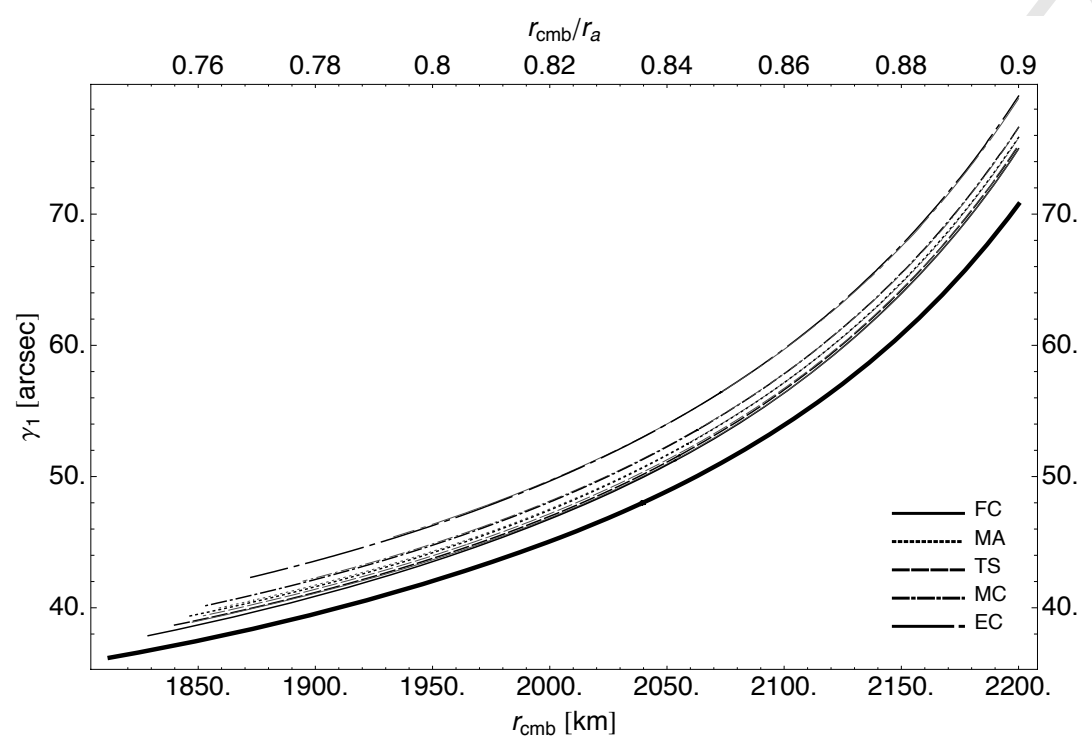

Fig. 7. 88-day libration amplitude as a function as a function of core size for hot (gray) and cold (black) models. Crust density is $2900 \mathrm{~kg} / \mathrm{m}^{3}$ except for the results for the cold FC model, which are also shown for the crust density of $3300 \mathrm{~kg} / \mathrm{m}^{3}$ (thick black). 


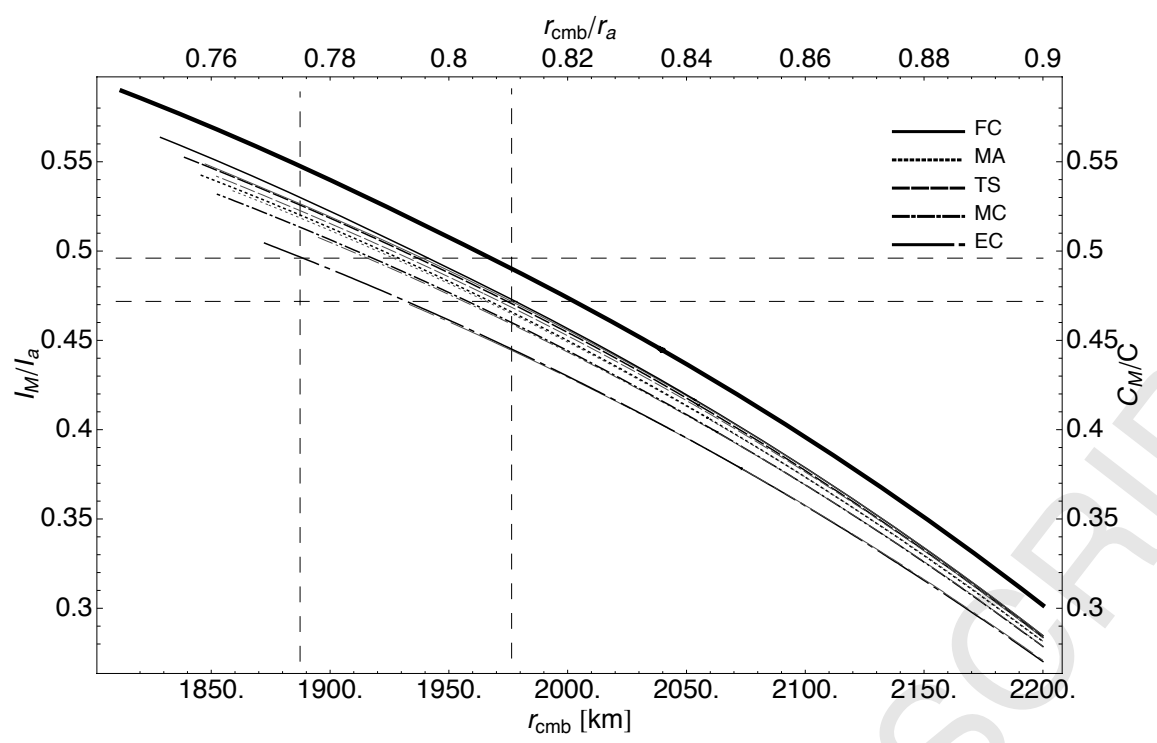

Fig. 8. Relative mantle moment as a function of core size for hot (gray) and cold (black) models. Crust density is $2900 \mathrm{~kg} / \mathrm{m}^{3}$ except for the results for the cold FC model, which are also shown for the crust density of $3300 \mathrm{~kg} / \mathrm{m}^{3}$ (thick black). 


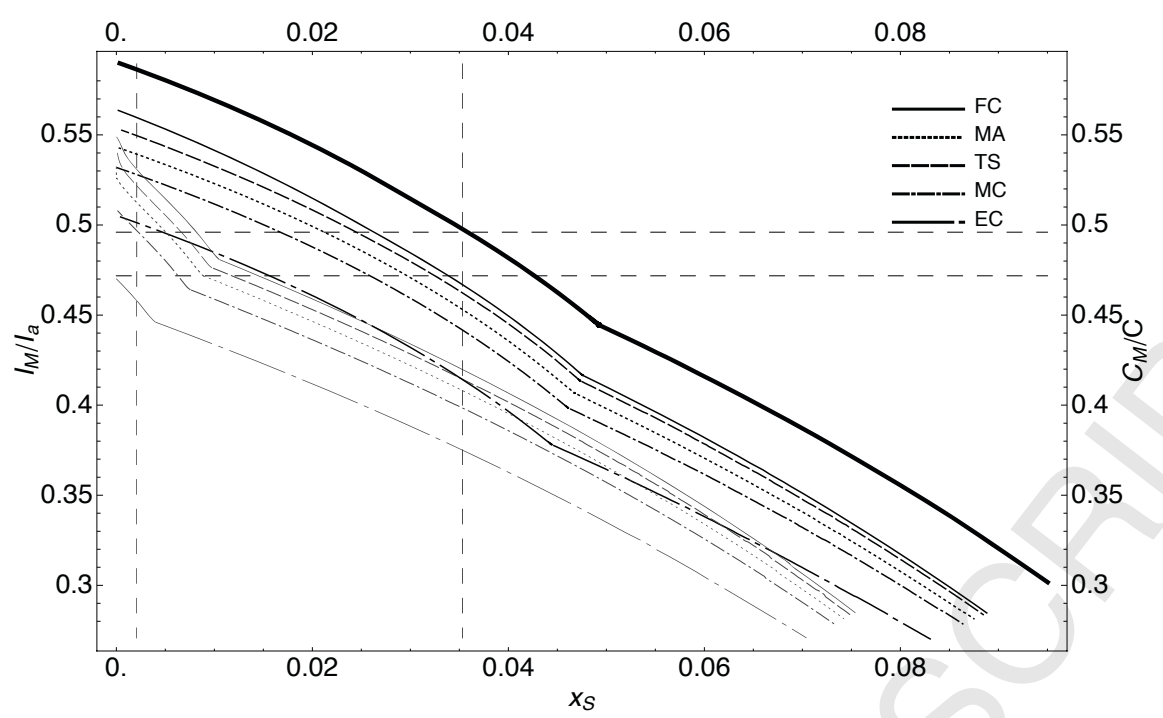

Fig. 9. Relative mantle moment as a function of core sulfur weight fraction for hot (gray) and cold (black) models. Crust density is $2900 \mathrm{~kg} / \mathrm{m}^{3}$ except for the results for the cold FC model, which are also shown for the crust density of $3300 \mathrm{~kg} / \mathrm{m}^{3}$ (thick black).

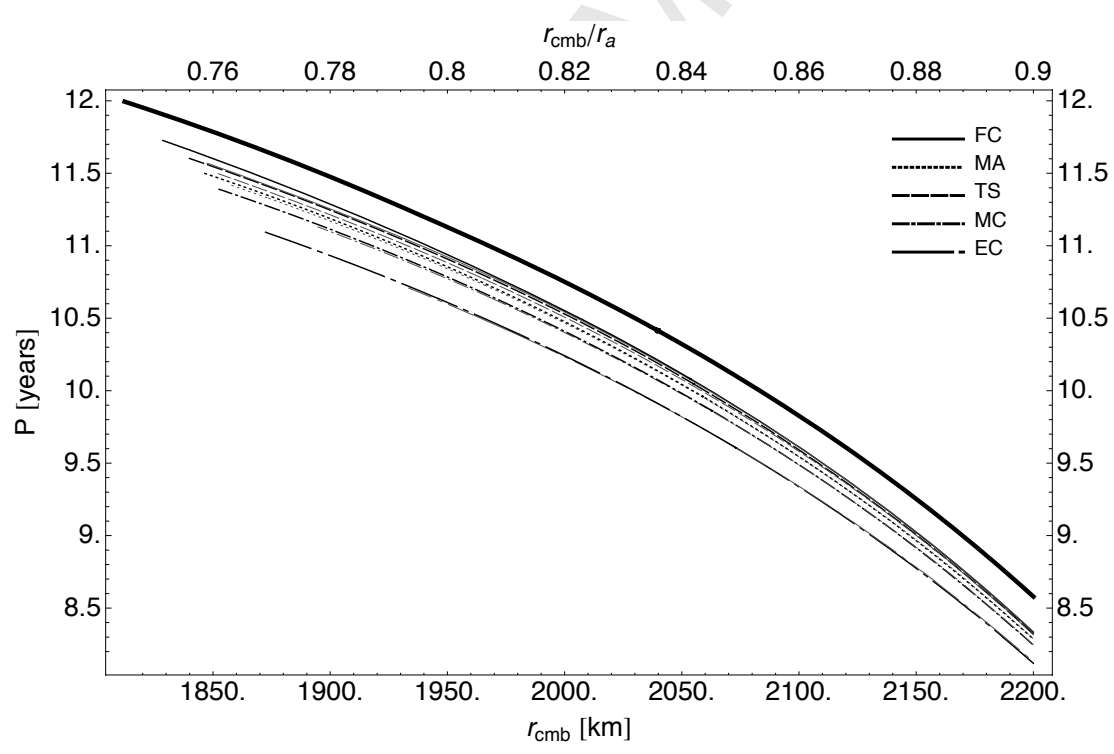

Fig. 10. Free period as a function as a function of core size for hot (gray) and cold (black) models. Crust density is $2900 \mathrm{~kg} / \mathrm{m}^{3}$ except for the results for the cold FC model, which are also shown for the crust density of $3300 \mathrm{~kg} / \mathrm{m}^{3}$ (thick black). 


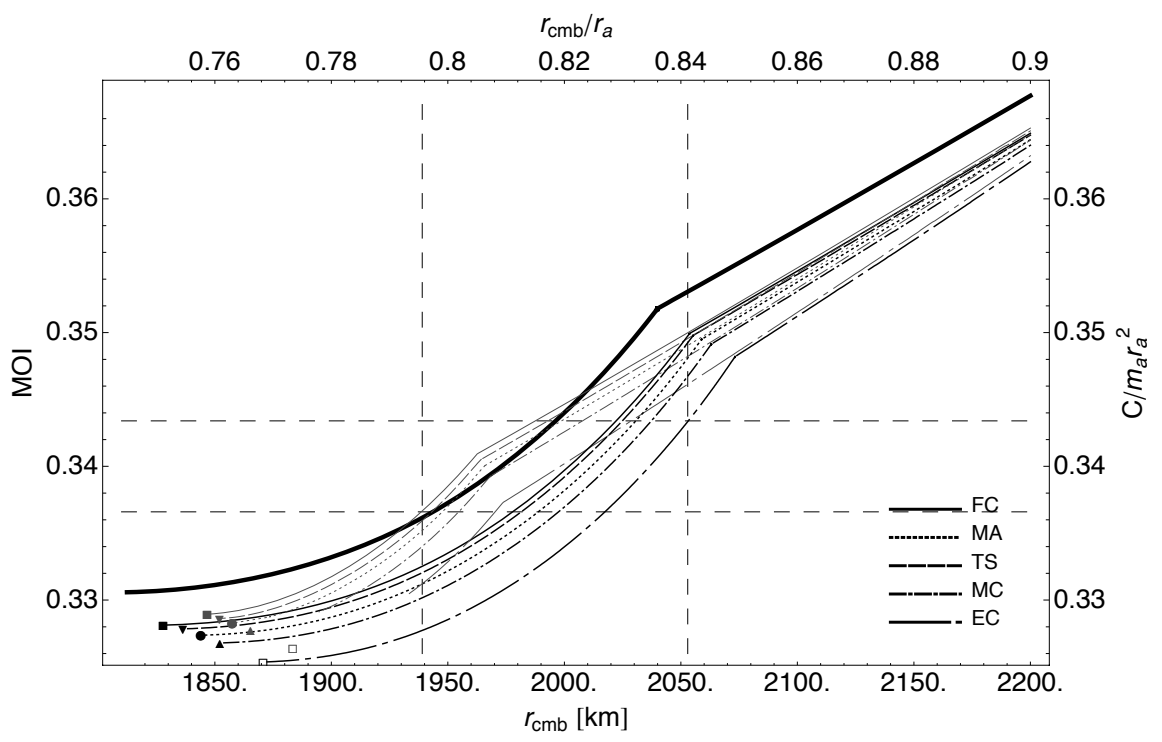

Fig. 11. MOI as a function of core size for hot (gray) and cold (black) models. Crust density is $2900 \mathrm{~kg} / \mathrm{m}^{3}$ except for the results for the cold FC model, which are also shown for the crust density of $3300 \mathrm{~kg} / \mathrm{m}^{3}$ (thick black).

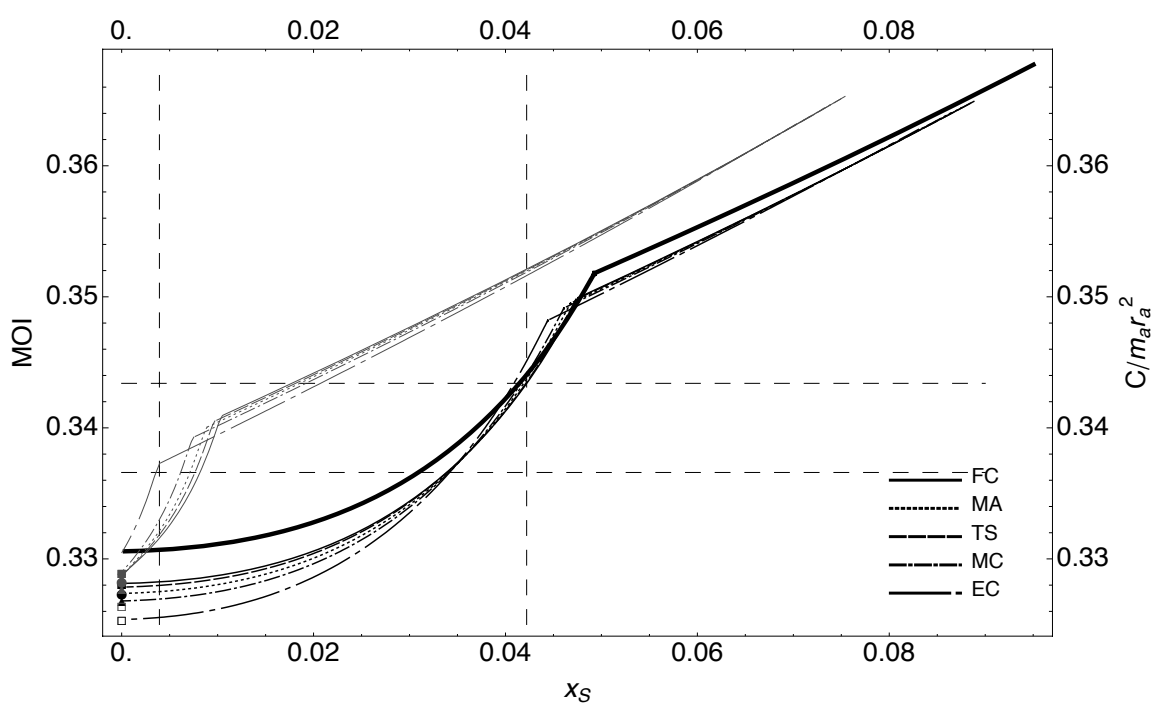

Fig. 12. MOI as a function of core sulfur weight fraction for hot (gray) and cold (black) models. Crust density is $2900 \mathrm{~kg} / \mathrm{m}^{3}$ except for the results for the cold FC model, which are also shown for the crust density of $3300 \mathrm{~kg} / \mathrm{m}^{3}$ (thick black). 


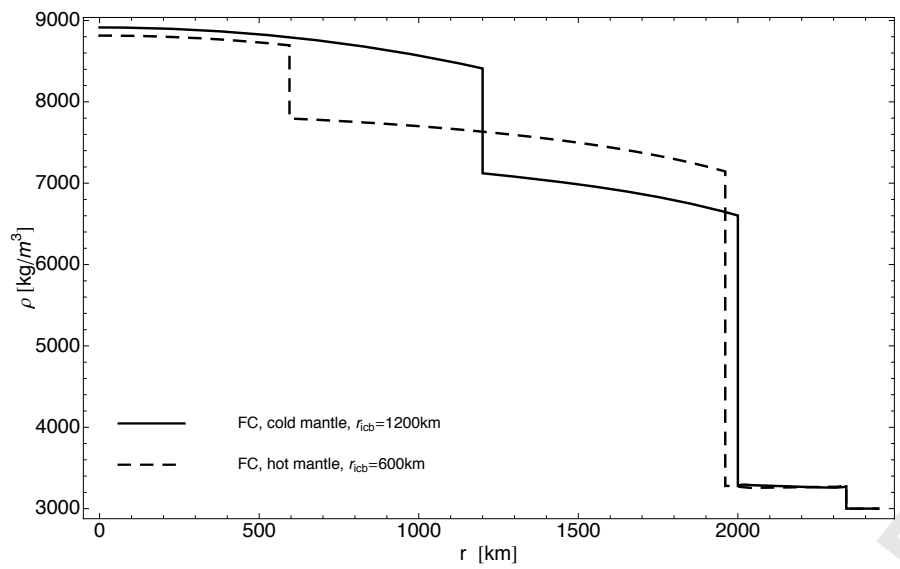

Fig. 13. Density profiles for hot (dashed) and cold (continuous) mantle temperatures.

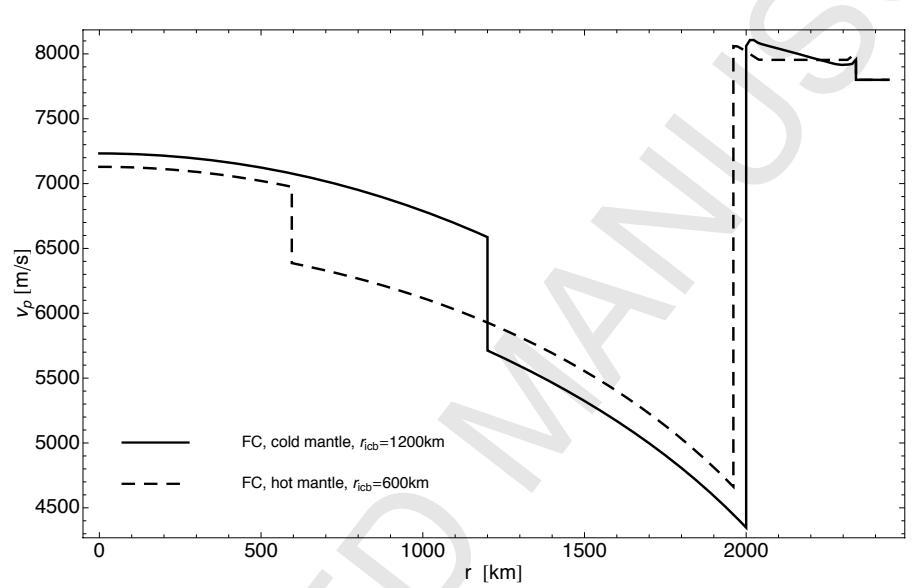

Fig. 14. $v_{p}$ profile for hot (dashed) and cold (continuous) mantle temperatures.

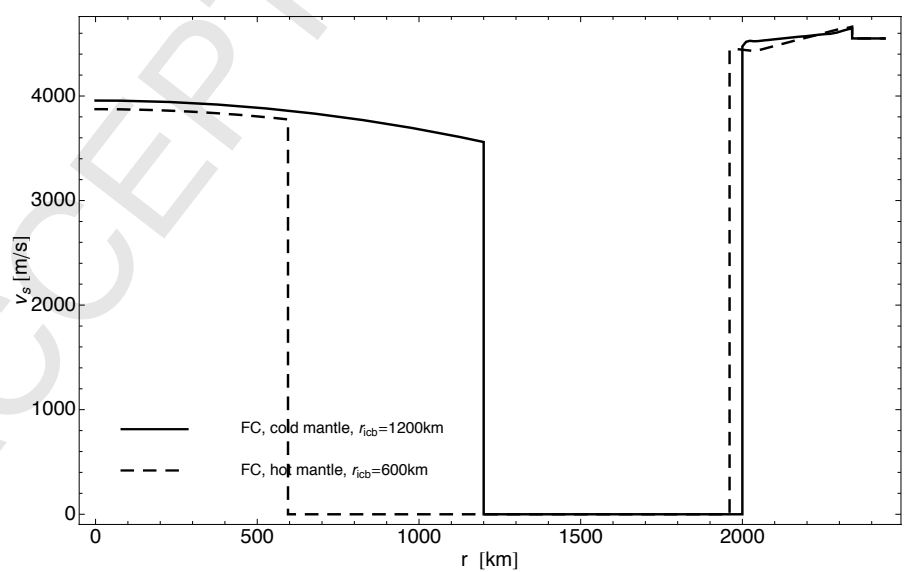

Fig. 15. $v_{s}$ profile for hot (dashed) and cold (continuous) mantle temperatures. 


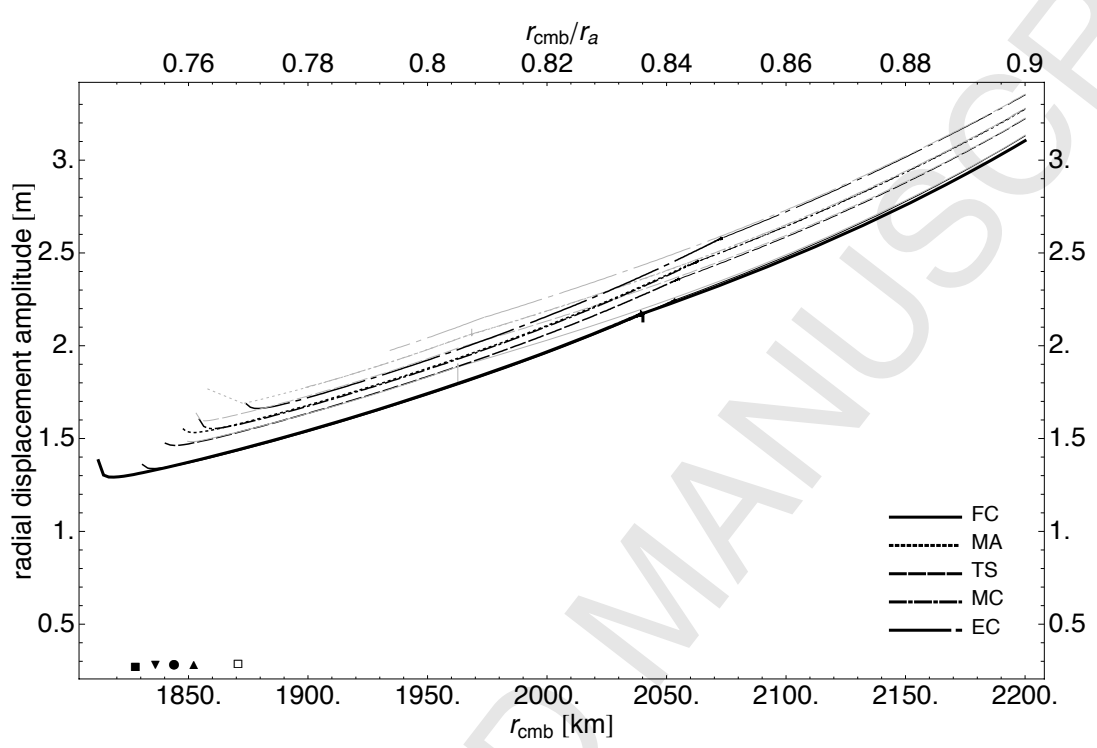

Fig. 16. Relative radial amplitude variations at the equator as a function of core size for hot (gray) and cold (black) models. Crust density is $2900 \mathrm{~kg} / \mathrm{m}^{3}$ except for the results for the cold FC model, which are also shown for the crust density of $3300 \mathrm{~kg} / \mathrm{m}^{3}$ (thick black). 


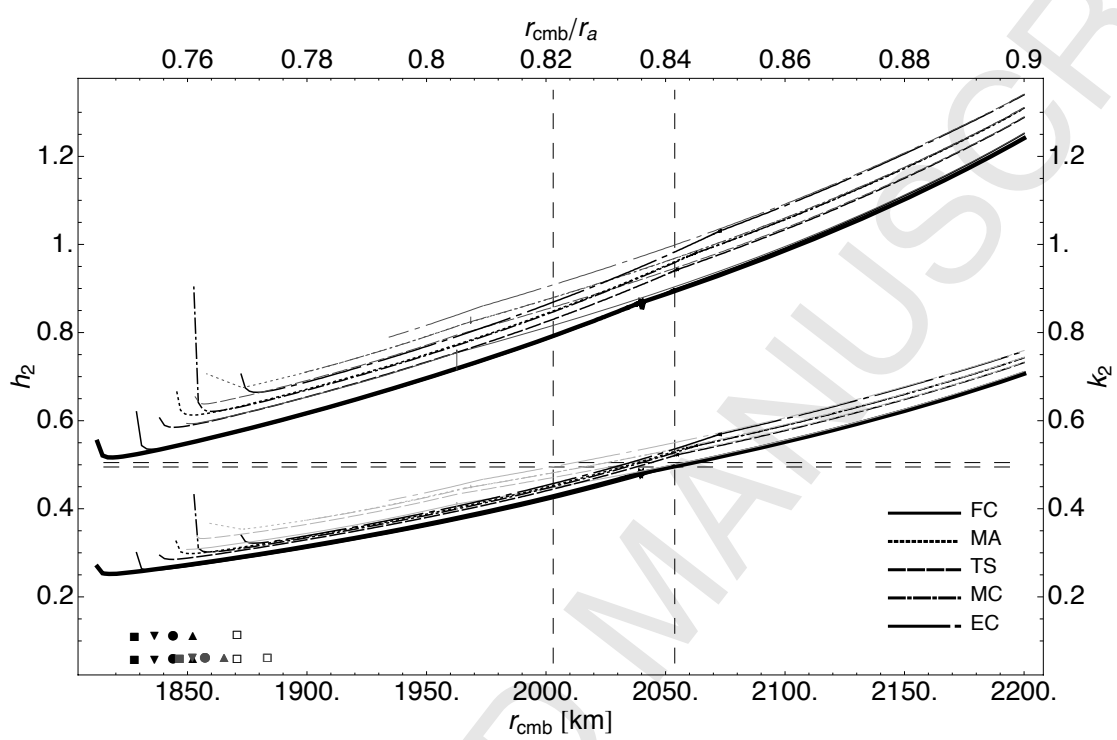

Fig. 17. Love numbers $h_{2}$ (upper curves) and $k_{2}$ (lower curves) as a function of core size for hot (gray) and cold (black) models. Crust density is $2900 \mathrm{~kg} / \mathrm{m}^{3}$ except for the results for the cold FC model, which are also shown for the crust density of $3300 \mathrm{~kg} / \mathrm{m}^{3}$ (thick black). 


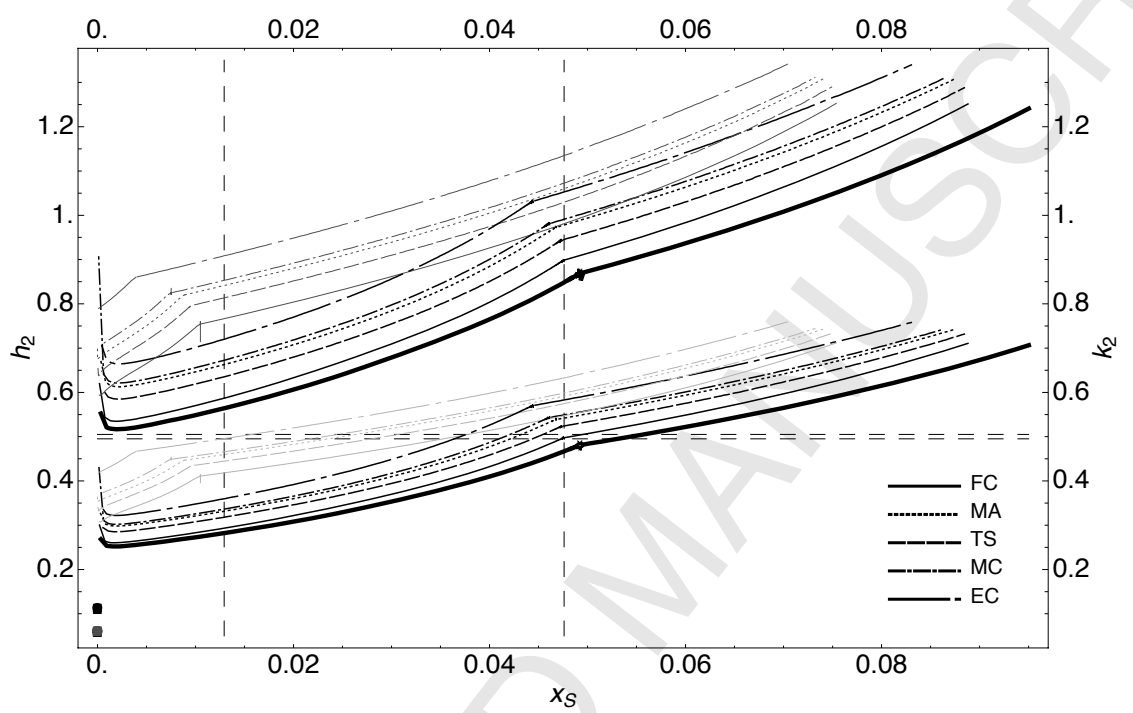

Fig. 18. Love numbers $h_{2}$ (upper curves) and $k_{2}$ (lower curves) as a function of core sulfur weight fraction for hot (gray) and cold (black) models. Crust density is $2900 \mathrm{~kg} / \mathrm{m}^{3}$ except for the results for the cold FC model, which are also shown for the crust density of $3300 \mathrm{~kg} / \mathrm{m}^{3}$ (thick black). 